\title{
Appendix 1. \\ Supplementary LA-ICP-MS data
}

Geochemical Sourcing of Lithic Raw Materials from Secondary Deposits in South Serbia. Implications for Early Neolithic Resource Management Strategies

\author{
Michael Brandl \\ Christoph Hauzenberger
}

The following LA-ICP-MS data tables contain the elemental concentration values of samples from Rasovača (RA), Kameno Rebro (KA), and Kremenac (KR), representing the primary geological sources, and Lebane (LEB) as test sample.

Three separate spots were analysed for each sample. All values are provided in parts per million (ppm). The data have not been logarithmically adjusted. 


\begin{tabular}{|c|c|c|c|c|c|c|c|c|c|c|}
\hline Sample No. & $\mathrm{Li7}$ & Be9 & B11 & Mg24 & $\mathrm{Al} 27$ & P31 & K39 & Ca43 & Ti49 & V51 \\
\hline RA1 & 62.7 & 2.02 & 39.2 & 48.4 & 8034.3 & 62.19 & 6999.9 & 442.0 & 399.64 & 218.58 \\
\hline RA1 & 72.9 & 2.78 & 41.0 & 51.0 & 7253.2 & 73.26 & 6240.9 & 385.7 & 463.99 & 236.26 \\
\hline RA1 & 64.4 & 2.48 & 37.8 & 48.3 & 11715.8 & 77.39 & 12603.6 & 423.5 & 465.81 & 217.64 \\
\hline RA2 & 4.6 & 1.03 & 21.1 & 1.7 & 384.0 & 30.09 & 128.2 & 106.4 & 0.98 & 3.13 \\
\hline RA2 & 4.8 & 4.05 & 44.0 & 4.2 & 507.3 & 90.89 & 92.9 & 275.4 & 18.03 & 25.85 \\
\hline RA2 & 5.2 & 4.85 & 39.2 & 15.0 & 527.8 & 102.86 & 90.6 & 263.8 & 14.55 & 32.30 \\
\hline RA3 & 56.4 & 4.55 & 65.2 & 128.7 & 10534.2 & 325.14 & 8020.1 & 415.8 & 671.30 & 532.16 \\
\hline RA3 & 67.2 & 0.83 & 12.5 & 228.2 & 26882.0 & 25.06 & 37551.9 & 244.5 & 155.67 & 56.10 \\
\hline RA3 & 53.4 & 0.89 & 21.4 & 214.1 & 37326.0 & 56.38 & 52994.9 & 340.2 & 391.75 & 121.08 \\
\hline RA4 & 35.9 & 1.80 & 40.1 & 98.6 & 9437.9 & 288.88 & 7091.3 & 517.7 & 474.86 & 168.13 \\
\hline RA4 & 38.2 & 1.22 & 36.8 & 108.9 & 16765.2 & 77.93 & 18511.4 & 522.7 & 564.91 & 158.07 \\
\hline RA4 & 35.8 & 1.53 & 42.4 & 84.0 & 14510.6 & 80.18 & 16030.4 & 503.0 & 559.95 & 192.05 \\
\hline RA5 & 42.1 & 2.56 & 45.1 & 628.2 & 6390.4 & 686.59 & 563.9 & 1945.8 & 94.32 & 87.75 \\
\hline RA5 & 36.1 & 1.92 & 52.5 & 40.0 & 3806.7 & 100.01 & 413.4 & 507.1 & 163.89 & 170.03 \\
\hline RA5 & 38.8 & 1.79 & 45.0 & 32.6 & 3776.4 & 83.56 & 435.3 & 526.3 & 107.09 & 137.65 \\
\hline RA6 & 5.3 & 1.49 & 31.8 & 14.3 & 1126.6 & 77.01 & 112.9 & 236.2 & 22.73 & 136.59 \\
\hline RA6 & 4.2 & 1.69 & 38.7 & 21.0 & 1421.1 & 116.12 & 106.5 & 330.9 & 22.70 & 188.52 \\
\hline RA6 & 4.7 & 1.32 & 39.0 & 24.3 & 1449.8 & 99.31 & 106.2 & 291.0 & 28.34 & 199.27 \\
\hline RA7 & 68.4 & 1.26 & 40.4 & 82.9 & 5683.0 & 65.50 & 2495.1 & 626.0 & 388.46 & 64.35 \\
\hline RA7 & 77.5 & 0.87 & 39.3 & 78.3 & 4874.3 & 56.38 & 1384.3 & 632.6 & 196.37 & 60.87 \\
\hline RA7 & 75.6 & 1.21 & 45.8 & 83.2 & 5292.2 & 86.49 & 1479.5 & 608.4 & 249.69 & 84.78 \\
\hline RA8 & 43.0 & 1.10 & 16.8 & 74.2 & 12459.0 & 1187.03 & 13352.7 & 889.2 & 188.00 & 31.23 \\
\hline RA8 & 42.8 & 1.62 & 12.2 & 75.9 & 15396.0 & 1845.13 & 15999.4 & 1341.5 & 116.76 & 16.62 \\
\hline RA8 & 54.7 & 1.89 & 37.7 & 64.3 & 9916.2 & 213.19 & 9367.6 & 563.8 & 377.32 & 139.78 \\
\hline RA9 & 12.1 & 2.29 & 45.9 & 12.9 & 1522.3 & 424.28 & 164.6 & 833.2 & 34.57 & 262.88 \\
\hline RA9 & 11.7 & 2.88 & 48.4 & 14.5 & 1735.3 & 1069.91 & 170.7 & 2453.1 & 35.09 & 309.55 \\
\hline RA9 & 10.3 & 2.06 & 45.1 & 12.5 & 1531.2 & 633.72 & 150.8 & 1560.4 & 32.80 & 284.53 \\
\hline RA10 & 9.3 & 0.70 & 44.3 & 8.7 & 725.0 & 186.18 & 232.7 & 328.0 & 10.17 & 18.54 \\
\hline RA10 & 21.0 & 1.25 & 42.1 & 10.0 & 1511.8 & 189.30 & 382.7 & 245.1 & 12.49 & 21.46 \\
\hline RA10 & 20.6 & 1.01 & 47.5 & 10.4 & 1462.3 & 139.09 & 360.5 & 231.6 & 18.68 & 20.37 \\
\hline RA11 & 64.2 & 1.73 & 48.1 & 60.4 & 8239.7 & 98.25 & 6375.4 & 494.1 & 389.84 & 197.71 \\
\hline RA11 & 83.5 & 2.47 & 44.6 & 45.0 & 5096.6 & 117.33 & 2542.7 & 289.9 & 287.17 & 173.29 \\
\hline RA11 & 57.9 & 1.33 & 19.5 & 73.5 & 7312.6 & 1608.67 & 1509.2 & 1788.2 & 82.37 & 16.53 \\
\hline RA12 & 2.2 & 1.68 & 37.6 & 12.0 & 713.2 & 106.03 & 98.6 & 179.3 & 1.04 & 96.46 \\
\hline RA12 & 1.5 & 2.18 & 48.5 & 23.7 & 945.3 & 125.45 & 87.6 & 329.9 & 0.95 & 163.52 \\
\hline RA12 & 2.0 & 1.76 & 46.4 & 17.2 & 917.5 & 102.68 & 112.0 & 179.1 & 1.81 & 125.52 \\
\hline RA13 & 59.6 & 1.96 & 34.6 & 65.4 & 13012.0 & 400.86 & 14427.8 & 733.0 & 475.46 & 129.50 \\
\hline RA13 & 86.8 & 0.73 & 15.0 & 38.2 & 6370.2 & 335.35 & 5441.9 & 660.3 & 159.41 & 34.41 \\
\hline RA13 & 92.3 & 2.11 & 24.7 & 34.7 & 7086.9 & 825.04 & 5910.2 & 1579.9 & 292.86 & 83.59 \\
\hline RA14 & 59.3 & 1.30 & 41.1 & 46.3 & 3027.6 & 57.19 & 304.6 & 369.2 & 16.12 & 208.60 \\
\hline RA14 & 49.0 & 1.44 & 55.6 & 59.1 & 3381.1 & 122.13 & 336.8 & 413.8 & 23.32 & 273.84 \\
\hline RA14 & 61.4 & 1.39 & 32.6 & 32.7 & 2819.2 & 92.13 & 251.6 & 302.4 & 16.12 & 148.63 \\
\hline RA15 & 6.1 & 1.41 & 31.4 & 13.7 & 539.9 & 160.93 & 82.7 & 187.0 & 0.75 & 123.10 \\
\hline RA15 & 4.5 & 1.69 & 29.9 & 20.7 & 474.5 & 177.90 & 67.6 & 202.4 & 0.72 & 113.49 \\
\hline RA15 & 4.7 & 2.79 & 32.2 & 15.3 & 687.5 & 205.94 & 96.5 & 267.6 & 1.00 & 164.28 \\
\hline
\end{tabular}




\begin{tabular}{|c|c|c|c|c|c|c|c|c|c|c|}
\hline Sample No. & Cr53 & Mn55 & Fe56 & Co59 & Ni60 & $\mathrm{Cu} 63$ & Zn66 & Ga71 & Ge74 & As75 \\
\hline RA1 & 3.37 & 165.9 & 142136 & 1.19 & 3.92 & 11.03 & 12.39 & 0.869 & 3.970 & 106.8 \\
\hline RA1 & 4.38 & 179.3 & 160466 & 1.44 & 4.38 & 13.85 & 14.37 & 1.109 & 5.030 & 114.2 \\
\hline RA1 & 3.89 & 169.8 & 145058 & 1.25 & 4.14 & 11.17 & 11.14 & 1.210 & 4.170 & 105.4 \\
\hline RA2 & 1.25 & 5.7 & 3949 & 0.07 & 0.45 & 1.63 & 0.59 & 0.599 & 3.030 & 21.4 \\
\hline RA2 & 0.89 & 28.3 & 34274 & 0.27 & 2.08 & 6.00 & 5.24 & 0.716 & 10.750 & 72.8 \\
\hline RA2 & 0.97 & 101.0 & 93874 & 5.73 & 6.08 & 5.77 & 6.67 & 0.817 & 10.670 & 239.9 \\
\hline RA3 & 7.60 & 380.6 & 375069 & 2.91 & 9.71 & 20.67 & 46.91 & 1.770 & 6.460 & 267.4 \\
\hline RA3 & 2.82 & 110.0 & 40082 & 2.01 & 2.99 & 9.17 & 6.91 & 2.710 & 2.420 & 26.2 \\
\hline RA3 & 3.04 & 154.0 & 93865 & 2.12 & 4.02 & 10.32 & 13.65 & 3.760 & 2.780 & 61.0 \\
\hline RA4 & 15.38 & 128.4 & 135525 & 1.55 & 6.98 & 16.21 & 18.03 & 1.510 & 3.890 & 79.5 \\
\hline RA4 & 11.04 & 127.3 & 139231 & 1.56 & 8.70 & 13.36 & 30.85 & 1.860 & 3.750 & 78.5 \\
\hline RA4 & 8.25 & 132.8 & 159764 & 1.44 & 7.29 & 14.58 & 47.22 & 1.830 & 4.280 & 93.4 \\
\hline RA5 & 5.88 & 101.0 & 96145 & 1.82 & 8.75 & 30.52 & 111.82 & 1.540 & 3.220 & 149.4 \\
\hline RA5 & 4.68 & 171.6 & 156703 & 4.45 & 15.08 & 11.00 & 20.45 & 0.595 & 3.510 & 197.3 \\
\hline RA5 & 3.73 & 148.8 & 128793 & 3.66 & 13.48 & 10.64 & 16.94 & 0.508 & 3.250 & 165.4 \\
\hline RA6 & 2.97 & 49.5 & 96650 & 4.16 & 13.93 & 4.32 & 7.99 & 0.593 & 2.690 & 133.8 \\
\hline RA6 & 2.79 & 64.2 & 130394 & 5.35 & 17.55 & 5.30 & 11.05 & 0.594 & 3.360 & 152.7 \\
\hline RA6 & 3.75 & 72.4 & 144294 & 5.42 & 18.39 & 5.10 & 12.79 & 0.701 & 3.180 & 167.0 \\
\hline RA7 & 4.94 & 198.0 & 116662 & 2.61 & 8.27 & 17.97 & 12.05 & 0.815 & 3.480 & 83.5 \\
\hline RA7 & 2.18 & 172.0 & 105267 & 2.56 & 8.34 & 17.17 & 10.93 & 0.611 & 3.710 & 72.4 \\
\hline RA7 & 3.98 & 215.2 & 135577 & 3.12 & 10.36 & 20.23 & 14.85 & 0.903 & 3.720 & 94.0 \\
\hline RA8 & 4.35 & 60.1 & 35771 & 0.44 & 1.92 & 25.45 & 23.46 & 1.380 & 2.400 & 48.5 \\
\hline RA8 & 6.41 & 38.9 & 18426 & 0.41 & 1.61 & 29.09 & 38.63 & 1.710 & 1.940 & 44.7 \\
\hline RA8 & 17.52 & 131.1 & 139340 & 0.94 & 3.70 & 12.95 & 12.90 & 1.300 & 4.380 & 101.5 \\
\hline RA9 & 1.00 & 84.2 & 177437 & 5.55 & 27.13 & 11.51 & 11.35 & 0.545 & 6.920 & 182.7 \\
\hline RA9 & 1.90 & 90.3 & 203741 & 6.43 & 33.01 & 11.95 & 13.17 & 0.507 & 7.810 & 206.4 \\
\hline RA9 & 1.61 & 83.8 & 186664 & 6.21 & 30.94 & 13.59 & 11.44 & 0.378 & 7.600 & 196.4 \\
\hline RA10 & 0.79 & 15.7 & 20456 & 1.38 & 3.39 & 4.95 & 5.38 & 0.426 & 1.730 & 144.9 \\
\hline RA10 & 0.94 & 20.6 & 26788 & 1.92 & 5.03 & 5.25 & 3.99 & 0.314 & 2.450 & 139.9 \\
\hline RA10 & 0.95 & 22.8 & 26504 & 1.58 & 4.74 & 3.35 & 3.97 & 0.491 & 3.070 & 143.4 \\
\hline RA11 & 3.41 & 203.5 & 153308 & 1.61 & 5.79 & 22.83 & 29.27 & 1.440 & 4.840 & 104.0 \\
\hline RA11 & 2.08 & 237.7 & 135935 & 1.36 & 5.63 & 16.49 & 13.87 & 1.190 & 4.430 & 103.8 \\
\hline RA11 & 4.54 & 57.3 & 22848 & 1.04 & 4.95 & 25.04 & 34.17 & 0.844 & 2.400 & 37.3 \\
\hline RA12 & 2.17 & 43.0 & 142500 & 3.44 & 27.24 & 11.16 & 6.47 & 0.963 & 7.650 & 238.2 \\
\hline RA12 & 1.56 & 81.0 & 242342 & 5.75 & 39.40 & 16.79 & 12.93 & 1.490 & 9.170 & 349.8 \\
\hline RA12 & 1.78 & 57.1 & 182897 & 4.71 & 34.07 & 12.92 & 7.91 & 1.042 & 8.160 & 254.7 \\
\hline RA13 & 10.72 & 141.3 & 88100 & 0.79 & 2.55 & 12.00 & 15.52 & 1.600 & 3.620 & 65.5 \\
\hline RA13 & 3.40 & 65.6 & 25252 & 0.43 & 1.59 & 9.52 & 13.52 & 0.792 & 2.800 & 29.5 \\
\hline RA13 & 2.36 & 106.4 & 54870 & 0.43 & 1.63 & 9.12 & 9.26 & 1.059 & 3.330 & 51.6 \\
\hline RA14 & 6.63 & 110.6 & 157928 & 3.86 & 20.78 & 6.80 & 24.13 & 1.390 & 5.310 & 84.3 \\
\hline RA14 & 11.30 & 155.1 & 218865 & 5.59 & 28.61 & 7.97 & 95.95 & 1.200 & 7.820 & 110.0 \\
\hline RA14 & 10.01 & 76.6 & 125206 & 3.19 & 17.48 & 6.34 & 20.36 & 0.352 & 3.430 & 68.8 \\
\hline RA15 & 1.37 & 109.6 & 133412 & 3.64 & 22.45 & 13.92 & 9.80 & 0.463 & 5.260 & 230.4 \\
\hline RA15 & 1.44 & 94.5 & 130013 & 3.45 & 22.53 & 17.55 & 20.93 & 0.661 & 4.370 & 187.7 \\
\hline RA15 & 1.60 & 128.0 & 201807 & 4.90 & 29.91 & 18.18 & 12.93 & 0.743 & 5.500 & 239.2 \\
\hline
\end{tabular}


$\begin{array}{lllllllllll}\text { Sample No. } & \text { Rb85 } & \text { Sr88 } & \text { Y89 } & \text { Zr90 } & \text { Nb93 } & \text { Cs133 } & \text { Ba137 } & \text { La139 } & \text { Ce140 } & \text { Pr141 }\end{array}$

\begin{tabular}{|c|c|c|c|c|c|c|c|c|c|c|}
\hline RA1 & 41.93 & 19.05 & 2.870 & 15.510 & 1.320 & 7.220 & 58.82 & 4.180 & 4.970 & 0.892 \\
\hline RA1 & 36.87 & 18.12 & 3.490 & 17.170 & 1.750 & 7.800 & 54.71 & 5.010 & 6.190 & 1.109 \\
\hline RA1 & 75.57 & 22.66 & 2.740 & 16.810 & 1.730 & 7.990 & 76.73 & 4.750 & 5.890 & 0.899 \\
\hline RA2 & 1.74 & 1.83 & 0.359 & 0.196 & 0.014 & 2.060 & 1.74 & 0.105 & 0.127 & 0.017 \\
\hline RA2 & 1.68 & 2.68 & 1.274 & 0.687 & 0.117 & 3.110 & 5.26 & 0.393 & 0.437 & 0.051 \\
\hline RA2 & 1.67 & 3.63 & 4.460 & 0.768 & 0.123 & 3.360 & 9.84 & 0.453 & 0.516 & 0.088 \\
\hline RA3 & 46.81 & 19.91 & 5.430 & 36.770 & 2.420 & 12.140 & 84.63 & 11.020 & 20.130 & 2.670 \\
\hline RA3 & 197.69 & 21.72 & 1.079 & 8.610 & 0.315 & 11.320 & 212.12 & 1.760 & 3.290 & 0.349 \\
\hline RA3 & 282.37 & 29.68 & 1.410 & 12.510 & 1.001 & 12.660 & 256.14 & 4.390 & 7.840 & 0.855 \\
\hline RA4 & 43.08 & 82.82 & 16.730 & 22.140 & 1.232 & 7.720 & 228.49 & 33.910 & 20.120 & 4.380 \\
\hline RA4 & 97.66 & 39.07 & 3.810 & 27.120 & 1.422 & 6.720 & 182.19 & 3.830 & 3.850 & 0.576 \\
\hline RA4 & 81.60 & 35.58 & 4.560 & 14.190 & 1.249 & 6.180 & 157.25 & 3.910 & 4.460 & 0.665 \\
\hline RA5 & 7.52 & 61.14 & 652.090 & 6.490 & 0.746 & 7.390 & 116.43 & 10.280 & 9.310 & 2.130 \\
\hline RA5 & 6.55 & 20.30 & 1.710 & 5.430 & 0.388 & 7.870 & 34.35 & 1.061 & 1.089 & 0.230 \\
\hline RA5 & 7.02 & 22.20 & 1.379 & 4.240 & 0.301 & 8.320 & 38.58 & 0.865 & 0.875 & 0.180 \\
\hline RA6 & 1.44 & 5.84 & 5.290 & 1.940 & 0.147 & 2.190 & 11.39 & 1.960 & 1.790 & 0.346 \\
\hline RA6 & 1.62 & 9.42 & 3.150 & 2.260 & 0.170 & 2.280 & 19.81 & 3.220 & 2.340 & 0.438 \\
\hline RA6 & 1.50 & 6.83 & 2.710 & 1.390 & 0.145 & 2.160 & 17.91 & 2.140 & 1.850 & 0.375 \\
\hline RA7 & 19.34 & 26.49 & 1.163 & 9.150 & 0.531 & 8.350 & 61.47 & 0.944 & 1.870 & 0.245 \\
\hline RA7 & 15.99 & 25.38 & 0.799 & 6.550 & 0.380 & 9.160 & 49.26 & 0.724 & 1.456 & 0.172 \\
\hline RA7 & 17.08 & 28.05 & 1.332 & 11.000 & 0.558 & 9.300 & 51.86 & 0.997 & 1.990 & 0.285 \\
\hline RA8 & 71.14 & 441.67 & 8.650 & 4.840 & 0.545 & 6.810 & 393.51 & 37.150 & 29.970 & 5.310 \\
\hline RA8 & 94.20 & 783.59 & 11.420 & 3.130 & 0.289 & 6.290 & 646.45 & 66.660 & 55.560 & 10.960 \\
\hline RA8 & 53.29 & 76.43 & 4.520 & 12.590 & 2.030 & 6.590 & 176.49 & 17.580 & 16.820 & 2.910 \\
\hline RA9 & 2.33 & 8.02 & 5.720 & 2.440 & 0.294 & 3.370 & 34.94 & 1.193 & 1.470 & 0.236 \\
\hline RA9 & 2.56 & 11.75 & 13.180 & 3.170 & 0.322 & 3.760 & 24.84 & 3.810 & 4.020 & 0.713 \\
\hline RA9 & 2.33 & 6.60 & 8.970 & 2.380 & 0.242 & 3.590 & 15.89 & 1.770 & 2.090 & 0.339 \\
\hline RA10 & 2.46 & 15.09 & 1.035 & 0.461 & 0.045 & 3.010 & 25.97 & 2.110 & 2.310 & 0.151 \\
\hline RA10 & 4.22 & 16.25 & 1.191 & 0.688 & 0.063 & 4.840 & 36.00 & 0.098 & 0.222 & 0.047 \\
\hline RA10 & 4.15 & 15.33 & 1.107 & 0.757 & 0.050 & 5.240 & 64.98 & 0.585 & 0.382 & 0.070 \\
\hline RA11 & 40.61 & 39.12 & 2.480 & 9.440 & 1.281 & 6.520 & 50.07 & 2.590 & 4.120 & 0.371 \\
\hline RA11 & 15.45 & 35.91 & 1.760 & 6.960 & 1.040 & 4.990 & 40.46 & 3.530 & 4.100 & 0.543 \\
\hline RA11 & 14.49 & 510.08 & 4.580 & 2.240 & 0.099 & 5.140 & 307.09 & 5.920 & 4.470 & 0.730 \\
\hline RA12 & 1.27 & 20.24 & 15.200 & 0.808 & 0.147 & 2.430 & 98.76 & 0.767 & 0.939 & 0.262 \\
\hline RA12 & 1.31 & 12.15 & 9.230 & 0.908 & 0.161 & 2.930 & 34.48 & 0.971 & 1.097 & 0.307 \\
\hline RA12 & 1.11 & 10.96 & 18.440 & 0.788 & 0.135 & 2.990 & 29.08 & 0.769 & 0.859 & 0.261 \\
\hline RA13 & 78.44 & 96.21 & 3.950 & 23.090 & 3.020 & 5.250 & 469.83 & 27.950 & 18.320 & 3.090 \\
\hline RA13 & 28.55 & 89.63 & 2.420 & 8.060 & 1.194 & 3.530 & 142.24 & 7.900 & 7.100 & 1.016 \\
\hline RA13 & 32.00 & 113.13 & 6.300 & 12.940 & 1.940 & 4.040 & 200.42 & 32.320 & 24.890 & 4.150 \\
\hline RA14 & 4.14 & 8.89 & 0.756 & 1.810 & 0.367 & 5.400 & 17.20 & 1.304 & 1.031 & 0.221 \\
\hline RA14 & 4.82 & 19.78 & 2.450 & 3.110 & 0.275 & 6.620 & 31.84 & 6.410 & 3.590 & 0.927 \\
\hline RA14 & 3.99 & 17.09 & 2.390 & 1.710 & 0.189 & 4.160 & 26.21 & 3.800 & 2.200 & 0.543 \\
\hline RA15 & 0.85 & 3.57 & 3.130 & 0.686 & 0.087 & 1.529 & 11.10 & 0.606 & 0.460 & 0.114 \\
\hline RA15 & 0.70 & 13.87 & 3.350 & 0.679 & 0.075 & 1.149 & 74.49 & 2.980 & 1.401 & 0.325 \\
\hline RA15 & 1.03 & 10.45 & 9.890 & 0.698 & 0.100 & 1.769 & 21.03 & 3.810 & 1.906 & 0.501 \\
\hline
\end{tabular}




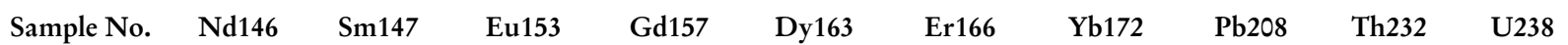

\begin{tabular}{|c|c|c|c|c|c|c|c|c|c|c|}
\hline RA1 & 3.390 & 0.810 & 0.359 & 0.705 & 0.727 & 0.341 & 0.352 & 46.29 & 1.360 & 0.703 \\
\hline RA1 & 4.320 & 1.054 & 0.412 & 0.890 & 0.791 & 0.460 & 0.445 & 52.63 & 1.334 & 0.825 \\
\hline RA1 & 3.280 & 0.736 & 0.294 & 0.611 & 0.627 & 0.337 & 0.341 & 62.19 & 1.770 & 0.736 \\
\hline RA2 & 0.054 & 0.084 & 0.022 & 0.062 & 0.067 & 0.060 & 0.059 & 1.34 & 0.016 & 0.050 \\
\hline RA2 & 0.205 & 0.072 & 0.067 & 0.149 & 0.145 & 0.140 & 0.202 & 9.23 & 0.053 & 0.173 \\
\hline RA2 & 0.395 & 0.116 & 0.066 & 0.143 & 0.462 & 0.708 & 0.738 & 17.00 & 0.062 & 0.257 \\
\hline RA3 & 9.840 & 1.670 & 0.370 & 1.350 & 1.030 & 0.599 & 0.586 & 306.23 & 1.930 & 1.770 \\
\hline RA3 & 1.320 & 0.212 & 0.074 & 0.188 & 0.218 & 0.137 & 0.159 & 10.84 & 1.208 & 0.160 \\
\hline RA3 & 3.130 & 0.467 & 0.110 & 0.327 & 0.285 & 0.142 & 0.078 & 50.87 & 2.360 & 0.282 \\
\hline RA4 & 12.630 & 1.790 & 0.400 & 1.570 & 1.810 & 1.142 & 1.083 & 314.10 & 4.600 & 1.470 \\
\hline RA4 & 2.090 & 0.498 & 0.144 & 0.348 & 0.436 & 0.450 & 0.660 & 100.66 & 4.180 & 0.980 \\
\hline RA4 & 2.180 & 0.467 & 0.138 & 0.483 & 0.555 & 0.423 & 0.491 & 116.76 & 2.550 & 0.934 \\
\hline RA5 & 11.040 & 5.730 & 2.760 & 23.050 & 35.920 & 30.610 & 23.730 & 97.57 & 1.580 & 2.900 \\
\hline RA5 & 0.905 & 0.300 & 0.054 & 0.145 & 0.212 & 0.163 & 0.254 & 61.62 & 0.678 & 1.180 \\
\hline RA5 & 0.697 & 0.157 & 0.074 & 0.147 & 0.154 & 0.154 & 0.193 & 47.72 & 0.589 & 1.006 \\
\hline RA6 & 1.092 & 0.329 & 0.126 & 0.381 & 0.428 & 0.395 & 0.489 & 32.61 & 0.191 & 0.729 \\
\hline RA6 & 1.390 & 0.309 & 0.163 & 0.308 & 0.241 & 0.266 & 0.327 & 50.08 & 0.173 & 0.899 \\
\hline RA6 & 1.190 & 0.261 & 0.145 & 0.205 & 0.232 & 0.231 & 0.280 & 49.97 & 0.191 & 1.036 \\
\hline RA7 & 0.716 & 0.217 & 0.059 & 0.160 & 0.176 & 0.144 & 0.207 & 42.61 & 1.390 & 0.306 \\
\hline RA7 & 0.792 & 0.150 & 0.074 & 0.090 & 0.144 & 0.083 & 0.112 & 35.95 & 0.920 & 0.274 \\
\hline RA7 & 1.048 & 0.211 & 0.085 & 0.236 & 0.173 & 0.153 & 0.161 & 52.02 & 1.223 & 0.397 \\
\hline RA8 & 19.320 & 3.120 & 0.705 & 2.630 & 1.720 & 0.683 & 0.498 & 823.76 & 1.179 & 0.288 \\
\hline RA8 & 37.680 & 6.190 & 1.259 & 4.490 & 2.500 & 0.882 & 0.408 & 606.96 & 0.734 & 0.212 \\
\hline RA8 & 10.100 & 1.550 & 0.466 & 1.350 & 0.896 & 0.418 & 0.384 & 221.73 & 1.540 & 0.606 \\
\hline RA9 & 1.012 & 0.206 & 0.117 & 0.455 & 0.519 & 0.518 & 0.771 & 32.72 & 0.235 & 1.590 \\
\hline RA9 & 3.390 & 0.733 & 0.320 & 0.975 & 1.260 & 1.370 & 1.720 & 39.95 & 0.349 & 1.820 \\
\hline RA9 & 1.340 & 0.414 & 0.228 & 0.596 & 0.760 & 0.729 & 0.945 & 31.63 & 0.215 & 1.570 \\
\hline RA10 & 0.359 & 0.062 & 0.018 & 0.078 & 0.082 & 0.088 & 0.079 & 16.17 & 0.177 & 0.148 \\
\hline RA10 & 0.127 & 0.083 & 0.019 & 0.048 & 0.062 & 0.074 & 0.122 & 8.71 & 0.103 & 0.202 \\
\hline RA10 & 0.307 & 0.101 & 0.022 & 0.062 & 0.163 & 0.118 & 0.119 & 8.76 & 0.105 & 0.171 \\
\hline RA11 & 1.134 & 0.135 & 0.102 & 0.186 & 0.258 & 0.236 & 0.370 & 109.75 & 1.240 & 0.882 \\
\hline RA11 & 1.500 & 0.203 & 0.117 & 0.251 & 0.202 & 0.211 & 0.259 & 90.93 & 0.881 & 0.697 \\
\hline RA11 & 2.430 & 0.529 & 0.175 & 0.411 & 0.509 & 0.457 & 0.409 & 529.89 & 0.283 & 0.150 \\
\hline RA12 & 1.580 & 0.698 & 0.170 & 1.310 & 1.740 & 1.520 & 1.570 & 63.77 & 0.018 & 2.030 \\
\hline RA12 & 1.750 & 0.635 & 0.149 & 0.732 & 1.050 & 0.977 & 1.260 & 59.01 & 0.020 & 3.620 \\
\hline RA12 & 1.690 & 0.850 & 0.256 & 1.660 & 1.940 & 1.670 & 1.990 & 43.34 & 0.011 & 2.720 \\
\hline RA13 & 9.270 & 1.470 & 0.419 & 1.230 & 0.783 & 0.514 & 0.561 & 105.71 & 2.660 & 0.764 \\
\hline RA13 & 3.220 & 0.496 & 0.161 & 0.490 & 0.494 & 0.311 & 0.254 & 50.12 & 3.310 & 0.266 \\
\hline RA13 & 13.270 & 2.350 & 0.670 & 1.630 & 1.280 & 0.586 & 0.604 & 50.96 & 1.880 & 0.799 \\
\hline RA14 & 0.786 & 0.119 & 0.046 & 0.196 & 0.121 & 0.086 & 0.123 & 21.33 & 0.278 & 0.648 \\
\hline RA14 & 3.160 & 0.452 & 0.123 & 0.318 & 0.353 & 0.232 & 0.250 & 48.91 & 0.660 & 1.110 \\
\hline RA14 & 2.000 & 0.309 & 0.092 & 0.273 & 0.341 & 0.314 & 0.374 & 37.33 & 0.389 & 0.557 \\
\hline RA15 & 0.498 & 0.153 & 0.052 & 0.141 & 0.253 & 0.313 & 0.343 & 19.92 & 0.010 & 1.640 \\
\hline RA15 & 1.203 & 0.201 & 0.091 & 0.325 & 0.359 & 0.328 & 0.495 & 21.35 & 0.012 & 1.468 \\
\hline RA15 & 1.790 & 0.475 & 0.119 & 0.765 & 1.135 & 0.970 & 1.259 & 35.59 & 0.012 & 2.100 \\
\hline
\end{tabular}




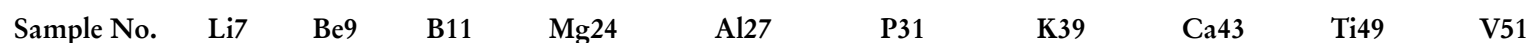

\begin{tabular}{|c|c|c|c|c|c|c|c|c|c|c|}
\hline KA1 & 0.6 & 0.28 & 26.9 & 5.9 & 47.2 & 15.13 & 26.6 & 81.9 & 2.14 & 0.14 \\
\hline KA1 & 0.4 & 0.32 & 27.8 & 5.4 & 45.9 & 16.06 & 31.9 & 79.0 & 0.65 & 0.07 \\
\hline KA1 & 0.3 & 0.24 & 32.0 & 4.7 & 36.7 & 10.53 & 24.9 & 71.9 & 1.50 & 0.04 \\
\hline KA2 & 0.5 & 0.53 & 26.0 & 6.5 & 66.2 & 13.97 & 36.9 & 85.6 & 0.75 & 0.06 \\
\hline KA2 & 0.3 & 0.35 & 32.6 & 5.2 & 55.0 & 15.85 & 29.6 & 84.0 & 0.64 & 0.17 \\
\hline KA2 & 0.3 & 0.19 & 34.1 & 4.3 & 36.5 & 16.10 & 28.0 & 84.1 & 1.57 & 0.11 \\
\hline KA3 & 1.1 & 2.20 & 49.8 & 4.9 & 70.9 & 17.52 & 70.8 & 74.5 & 1.53 & 0.65 \\
\hline KA3 & 0.8 & 1.75 & 45.2 & 4.4 & 63.4 & 19.40 & 62.5 & 74.2 & 1.79 & 0.80 \\
\hline KA3 & 0.8 & 1.47 & 41.2 & 4.4 & 67.2 & 23.67 & 49.5 & 68.7 & 46.95 & 30.98 \\
\hline KA4 & 7.3 & 5.97 & 34.6 & 6.8 & 213.6 & 20.65 & 154.7 & 69.2 & 1.24 & 0.49 \\
\hline KA4 & 4.6 & 7.77 & 33.4 & 6.4 & 116.1 & 14.60 & 108.2 & 63.0 & 1.35 & 0.47 \\
\hline KA4 & 13.0 & 6.36 & 34.8 & 12.0 & 396.9 & 16.51 & 258.1 & 83.8 & 81.85 & 12.38 \\
\hline KA5 & 3.7 & 1.37 & 29.5 & 9.4 & 197.4 & 31.88 & 106.7 & 85.9 & 1.35 & 13.29 \\
\hline KA5 & 6.0 & 2.40 & 30.3 & 20.6 & 398.7 & 143.71 & 170.1 & 125.8 & 8.78 & 94.69 \\
\hline KA5 & 7.5 & 1.33 & 33.4 & 16.3 & 444.5 & 74.23 & 206.2 & 67.3 & 4.67 & 41.99 \\
\hline KA6 & 0.6 & 0.31 & 18.6 & 10.2 & 53.5 & 36.06 & 94.6 & 166.4 & 3.67 & 2.19 \\
\hline KA6 & 0.4 & 0.21 & 13.7 & 8.8 & 44.0 & 16.85 & 77.6 & 118.4 & 1.56 & 2.22 \\
\hline KA6 & 0.4 & 0.22 & 12.8 & 3.2 & 43.6 & 27.73 & 74.1 & 119.9 & 1.44 & 2.06 \\
\hline KA7 & 0.4 & 0.32 & 36.1 & 3.9 & 28.7 & 17.83 & 28.1 & 91.8 & 1.12 & 0.05 \\
\hline KA7 & 0.5 & 0.27 & 31.5 & 4.3 & 32.3 & 21.85 & 25.6 & 98.9 & 0.71 & 0.14 \\
\hline KA7 & 0.3 & 0.25 & 33.5 & 4.7 & 39.2 & 14.35 & 27.0 & 89.8 & 1.40 & 0.12 \\
\hline KA8 & 1.1 & 2.80 & 55.0 & 4.9 & 58.3 & 15.56 & 105.9 & 78.0 & 1.08 & 0.74 \\
\hline KA8 & 2.3 & 1.94 & 54.2 & 5.2 & 189.4 & 21.16 & 139.2 & 81.9 & 2.00 & 0.65 \\
\hline KA8 & 5.6 & 1.98 & 35.9 & 5.8 & 295.9 & 18.06 & 160.6 & 72.3 & 1.69 & 0.59 \\
\hline KA9 & 0.9 & 0.18 & 27.0 & 3.8 & 69.6 & 19.19 & 42.0 & 88.0 & 2.09 & 0.10 \\
\hline KA9 & 0.9 & 0.20 & 29.0 & 4.6 & 88.7 & 19.28 & 42.3 & 86.3 & 2.39 & 0.10 \\
\hline KA9 & 1.4 & 0.30 & 25.5 & 7.4 & 68.2 & 16.77 & 39.2 & 87.5 & 2.19 & 0.12 \\
\hline KA10 & 1.0 & 0.96 & 30.8 & 4.2 & 59.0 & 16.22 & 51.4 & 83.9 & 3.32 & 0.62 \\
\hline KA10 & 1.1 & 0.63 & 28.7 & 4.1 & 75.2 & 19.16 & 54.5 & 81.2 & 1.81 & 0.17 \\
\hline KA10 & 0.9 & 0.33 & 27.8 & 4.5 & 104.5 & 22.38 & 61.5 & 99.7 & 1.98 & 0.15 \\
\hline KA11 & 1.4 & 1.85 & 55.3 & 4.0 & 46.3 & 21.48 & 87.8 & 73.7 & 1.94 & 1.44 \\
\hline KA11 & 1.6 & 2.63 & 59.0 & 4.1 & 75.5 & 11.43 & 92.0 & 75.9 & 1.78 & 1.28 \\
\hline KA11 & 1.5 & 1.61 & 51.7 & 3.6 & 43.1 & 15.90 & 82.4 & 85.6 & 2.14 & 1.45 \\
\hline KA12 & 3.9 & 0.68 & 32.5 & 7.8 & 322.8 & 13.13 & 164.7 & 133.2 & 6.52 & 0.40 \\
\hline KA12 & 4.4 & 0.57 & 29.6 & 12.7 & 307.4 & 11.99 & 178.9 & 91.6 & 5.22 & 0.26 \\
\hline KA12 & 2.5 & 0.63 & 37.1 & 6.3 & 207.4 & 13.37 & 105.3 & 107.2 & 4.24 & 0.28 \\
\hline KA13 & 1.0 & 0.41 & 36.3 & 5.0 & 75.6 & 11.38 & 62.9 & 85.8 & 0.64 & 0.19 \\
\hline KA13 & 1.6 & 0.44 & 27.1 & 5.9 & 122.1 & 23.96 & 77.9 & 98.4 & 0.79 & 0.29 \\
\hline KA13 & 3.1 & 0.50 & 32.6 & 6.9 & 177.6 & 18.60 & 98.9 & 98.8 & 0.75 & 0.31 \\
\hline KA14 & 2.7 & 3.92 & 42.9 & 5.9 & 139.7 & 15.02 & 112.6 & 78.3 & 0.81 & 0.39 \\
\hline KA14 & 3.2 & 3.44 & 47.6 & 7.6 & 202.5 & 19.37 & 155.9 & 75.0 & 1.29 & 0.41 \\
\hline KA14 & 3.8 & 3.75 & 48.5 & 7.0 & 215.2 & 15.18 & 161.9 & 104.1 & 0.72 & 0.50 \\
\hline KA15 & 28.0 & 2.45 & 32.5 & 6.3 & 614.4 & 19.90 & 286.3 & 82.3 & 1.53 & 0.84 \\
\hline KA15 & 4.3 & 4.20 & 38.7 & 6.9 & 203.7 & 15.51 & 128.7 & 83.0 & 6.22 & 2.26 \\
\hline KA15 & 50.6 & 5.57 & 34.9 & 5.2 & 746.6 & 9.75 & 330.9 & 88.8 & 0.68 & 0.71 \\
\hline
\end{tabular}




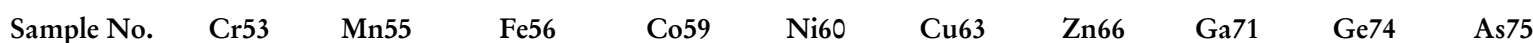

\begin{tabular}{|c|c|c|c|c|c|c|c|c|c|c|}
\hline KA1 & 1.14 & 0.8 & 20 & 0.03 & 0.15 & 0.27 & 0.48 & 0.122 & 6.730 & 46.3 \\
\hline KA1 & 1.18 & 0.4 & 15 & 0.04 & 0.13 & 0.26 & 0.31 & 0.132 & 5.760 & 42.1 \\
\hline KA1 & 1.11 & 0.4 & 10 & 0.03 & 0.24 & 0.24 & 0.50 & 0.062 & 3.970 & 39.2 \\
\hline KA2 & 1.13 & 1.0 & 24.9 & 0.03 & 0.14 & 0.28 & 0.75 & 0.209 & 4.030 & 28.2 \\
\hline KA2 & 1.10 & 0.7 & 30.7 & 0.04 & 0.16 & 0.24 & 0.56 & 0.267 & 3.540 & 32.1 \\
\hline KA2 & 1.25 & 0.5 & 9.7 & 0.03 & 0.17 & 0.27 & 0.32 & 0.198 & 3.460 & 43.1 \\
\hline KA3 & 0.97 & 1.1 & 85 & 0.03 & 0.25 & 0.37 & 0.56 & 0.535 & 5.130 & 85.5 \\
\hline KA3 & 0.91 & 0.6 & 82 & 0.03 & 0.15 & 0.20 & 0.60 & 0.618 & 5.890 & 92.3 \\
\hline KA3 & 17.13 & 1.0 & 167 & 0.05 & 0.21 & 0.34 & 0.37 & 1.452 & 5.970 & 85.2 \\
\hline KA4 & 0.87 & 1.1 & 102.4 & 0.02 & 0.24 & 0.22 & 0.72 & 1.680 & 4.640 & 57.6 \\
\hline KA4 & 0.83 & 1.2 & 149.1 & 0.03 & 0.24 & 0.71 & 0.58 & 2.090 & 6.010 & 56.3 \\
\hline KA4 & 1.52 & 1.7 & 180.7 & 0.03 & 0.34 & 0.38 & 0.58 & 1.820 & 5.430 & 58.7 \\
\hline KA5 & 1.19 & 10.5 & 3703 & 0.04 & 0.72 & 0.29 & 1.07 & 0.062 & 3.530 & 63.6 \\
\hline KA5 & 0.63 & 17.1 & 23407 & 0.24 & 3.33 & 1.39 & 4.31 & 0.081 & 8.080 & 144.6 \\
\hline KA5 & 0.82 & 7.5 & 11567 & 0.17 & 1.94 & 0.53 & 2.67 & 0.078 & 4.890 & 95.1 \\
\hline KA6 & 2.23 & 1.1 & 47.4 & 0.06 & 0.28 & 0.54 & 2.39 & 0.140 & 1.330 & 5.8 \\
\hline KA6 & 1.53 & 0.8 & 55.7 & 0.04 & 0.31 & 0.37 & 0.58 & 0.078 & 1.110 & 7.6 \\
\hline KA6 & 1.46 & 0.5 & 35.6 & 0.05 & 0.42 & 0.40 & 0.85 & 0.082 & 1.230 & 7.7 \\
\hline KA7 & 1.17 & 0.7 & 11 & 0.03 & 0.12 & 0.32 & 0.36 & 0.118 & 5.770 & 44.1 \\
\hline KA7 & 1.36 & 0.8 & 11 & 0.04 & 0.21 & 0.30 & 0.66 & 0.088 & 4.200 & 38.4 \\
\hline KA7 & 1.26 & 0.7 & 19 & 0.04 & 0.15 & 0.29 & 0.45 & 0.111 & 3.940 & 43.0 \\
\hline KA8 & 0.81 & 1.8 & 186.4 & 0.03 & 0.13 & 0.19 & 0.51 & 1.930 & 9.390 & 101.4 \\
\hline KA8 & 1.07 & 2.2 & 71.9 & 0.03 & 0.20 & 0.27 & 0.30 & 1.344 & 4.280 & 85.7 \\
\hline KA8 & 0.90 & 1.4 & 93.0 & 0.03 & 0.11 & 0.24 & 0.35 & 1.018 & 5.670 & 79.9 \\
\hline KA9 & 1.18 & 1.1 & 18 & 0.04 & 0.36 & 0.28 & 0.29 & 0.185 & 2.530 & 33.0 \\
\hline KA9 & 1.02 & 0.5 & 18 & 0.03 & 0.12 & 0.28 & 0.33 & 0.151 & 2.090 & 35.7 \\
\hline KA9 & 1.15 & 40.4 & 59 & 0.29 & 1.58 & 0.30 & 0.54 & 0.107 & 6.250 & 31.0 \\
\hline KA10 & 1.00 & 1.0 & 143.2 & 0.06 & 0.50 & 0.50 & 0.41 & 0.079 & 7.990 & 43.8 \\
\hline KA10 & 1.18 & 0.8 & 59.8 & 0.03 & 0.26 & 0.27 & 0.62 & 0.101 & 7.360 & 34.7 \\
\hline KA10 & 1.16 & 0.8 & 48.8 & 0.04 & 0.42 & 0.32 & 0.56 & 0.086 & 5.050 & 36.1 \\
\hline KA11 & 1.05 & 1.0 & 87 & 0.02 & 0.09 & 0.25 & 0.45 & 0.341 & 5.440 & 98.7 \\
\hline KA11 & 0.95 & 0.9 & 74 & 0.04 & 0.12 & 0.25 & 0.59 & 0.422 & 5.410 & 105.5 \\
\hline KA11 & 1.15 & 0.9 & 88 & 0.03 & 0.28 & 0.25 & 0.58 & 0.361 & 6.840 & 113.7 \\
\hline KA12 & 1.24 & 0.3 & 103.2 & 0.04 & 0.17 & 0.30 & 0.62 & 0.073 & 4.360 & 46.6 \\
\hline KA12 & 1.10 & 0.4 & 82.0 & 0.04 & 0.23 & 0.32 & 0.43 & 0.164 & 5.050 & 43.0 \\
\hline KA12 & 1.39 & 0.4 & 100.9 & 0.04 & 0.19 & 0.34 & 0.85 & 0.208 & 3.930 & 52.4 \\
\hline KA13 & 1.22 & 0.6 & 48 & 0.03 & 0.15 & 0.31 & 0.37 & 0.066 & 3.200 & 40.1 \\
\hline KA13 & 1.29 & 0.8 & 54 & 0.05 & 0.21 & 0.28 & 0.36 & 0.072 & 5.170 & 33.4 \\
\hline KA13 & 1.15 & 0.9 & 63 & 0.03 & 0.20 & 0.29 & 2.09 & 0.137 & 3.720 & 34.7 \\
\hline KA14 & 1.01 & 1.2 & 74.1 & 0.03 & 0.23 & 0.28 & 0.34 & 0.710 & 5.070 & 77.4 \\
\hline KA14 & 1.06 & 1.1 & 112.0 & 0.03 & 0.24 & 0.39 & 0.37 & 0.747 & 5.500 & 82.0 \\
\hline KA14 & 1.12 & 1.1 & 102.5 & 0.03 & 0.16 & 0.30 & 0.34 & 0.662 & 6.570 & 81.0 \\
\hline KA15 & 1.08 & 6.5 & 73 & 0.09 & 0.19 & 0.30 & 0.30 & 1.021 & 4.110 & 50.7 \\
\hline KA15 & 1.01 & 3.0 & 91 & 0.04 & 0.13 & 0.26 & 0.28 & 1.260 & 4.410 & 72.6 \\
\hline KA15 & 0.97 & 100.1 & 120 & 2.49 & 0.70 & 0.33 & 0.59 & 2.370 & 5.450 & 47.7 \\
\hline
\end{tabular}


$\begin{array}{lllllllllll}\text { Sample No. } & \text { Rb85 } & \text { Sr88 } & \text { Y89 } & \text { Zr90 } & \text { Nb93 } & \text { Cs133 } & \text { Ba137 } & \text { La139 } & \text { Ce140 } & \text { Pr141 }\end{array}$

\begin{tabular}{|c|c|c|c|c|c|c|c|c|c|c|}
\hline KA1 & 0.23 & 0.29 & 0.010 & 0.266 & 0.226 & 0.279 & 1.64 & 0.017 & 0.031 & 0.007 \\
\hline KA1 & 0.20 & 0.23 & 0.012 & 0.153 & 0.206 & 0.303 & 1.09 & 0.007 & 0.011 & 0.009 \\
\hline KA1 & 0.20 & 0.28 & 0.014 & 0.226 & 0.161 & 0.145 & 1.60 & 0.010 & 0.008 & 0.004 \\
\hline KA2 & 0.29 & 0.26 & 0.007 & 0.678 & 0.315 & 0.314 & 1.62 & 0.009 & 0.005 & 0.009 \\
\hline KA2 & 0.40 & 0.25 & 0.018 & 0.658 & 0.270 & 0.204 & 1.74 & 0.016 & 0.049 & 0.006 \\
\hline KA2 & 0.32 & 0.28 & 0.012 & 0.443 & 0.255 & 0.229 & 1.66 & 0.014 & 0.015 & 0.011 \\
\hline KA3 & 0.75 & 0.59 & 0.045 & 0.192 & 0.128 & 1.333 & 2.95 & 0.073 & 0.085 & 0.015 \\
\hline KA3 & 0.68 & 0.56 & 1.370 & 0.147 & 0.109 & 1.306 & 3.79 & 0.082 & 0.146 & 0.017 \\
\hline KA3 & 0.55 & 0.56 & 4.270 & 0.177 & 0.226 & 1.012 & 7.84 & 0.199 & 0.307 & 0.045 \\
\hline KA4 & 1.62 & 1.09 & 0.055 & 0.159 & 0.016 & 3.100 & 8.26 & 0.038 & 0.032 & 0.008 \\
\hline KA4 & 1.18 & 1.25 & 0.039 & 0.172 & 0.010 & 2.160 & 18.69 & 0.059 & 0.058 & 0.010 \\
\hline KA4 & 2.53 & 1.29 & 0.169 & 0.583 & 0.174 & 4.870 & 4.88 & 0.331 & 0.852 & 0.058 \\
\hline KA5 & 1.19 & 0.67 & 0.110 & 0.330 & 0.026 & 1.101 & 6.58 & 0.024 & 0.071 & 0.011 \\
\hline KA5 & 2.05 & 1.44 & 5.860 & 0.818 & 0.142 & 1.990 & 11.26 & 0.306 & 0.981 & 0.135 \\
\hline KA5 & 2.44 & 1.13 & 3.840 & 0.587 & 0.035 & 2.280 & 5.77 & 0.200 & 0.497 & 0.086 \\
\hline KA6 & 0.46 & 0.40 & 0.017 & 0.182 & 0.107 & 0.233 & 2.71 & 0.024 & 0.083 & 0.013 \\
\hline KA6 & 0.53 & 0.21 & 0.024 & 0.145 & 0.050 & 0.159 & 1.20 & 0.015 & 0.014 & 0.014 \\
\hline KA6 & 0.52 & 0.38 & 0.018 & 0.153 & 0.059 & 0.176 & 2.57 & 0.013 & 0.038 & 0.088 \\
\hline KA7 & 0.26 & 0.22 & 0.014 & 0.060 & 0.312 & 0.158 & 1.23 & 0.014 & 0.035 & 0.014 \\
\hline KA7 & 0.24 & 0.26 & 0.095 & 0.062 & 0.303 & 0.139 & 1.08 & 0.014 & 0.015 & 0.007 \\
\hline KA7 & 0.32 & 0.25 & 0.056 & 0.098 & 0.384 & 0.270 & 1.32 & 0.021 & 0.071 & 0.011 \\
\hline KA8 & 1.09 & 0.80 & 0.036 & 0.067 & 0.141 & 2.150 & 1.69 & 0.025 & 0.017 & 0.006 \\
\hline KA8 & 1.27 & 0.87 & 0.026 & 0.193 & 0.147 & 2.480 & 3.18 & 0.023 & 0.012 & 0.006 \\
\hline KA8 & 1.64 & 0.78 & 0.036 & 0.389 & 0.160 & 2.980 & 2.40 & 0.020 & 0.009 & 0.004 \\
\hline KA9 & 0.46 & 0.24 & 0.013 & 0.703 & 0.220 & 0.451 & 1.88 & 0.016 & 0.010 & 0.011 \\
\hline KA9 & 0.49 & 0.28 & 0.016 & 0.920 & 0.219 & 0.462 & 1.83 & 0.021 & 0.041 & 0.012 \\
\hline KA9 & 0.31 & 0.45 & 0.051 & 0.533 & 0.132 & 0.414 & 2.77 & 0.025 & 0.046 & 0.010 \\
\hline KA10 & 0.51 & 0.46 & 0.074 & 0.423 & 0.064 & 0.460 & 3.62 & 0.049 & 0.179 & 0.018 \\
\hline KA10 & 0.49 & 0.22 & 0.033 & 0.482 & 0.031 & 0.502 & 1.18 & 0.027 & 0.082 & 0.013 \\
\hline KA10 & 0.69 & 0.38 & 0.038 & 0.552 & 0.125 & 0.447 & 3.23 & 0.050 & 0.190 & 0.017 \\
\hline KA11 & 0.75 & 0.50 & 0.101 & 0.052 & 0.014 & 1.780 & 1.62 & 0.025 & 0.008 & 0.004 \\
\hline KA11 & 0.71 & 0.44 & 0.018 & 0.114 & 0.037 & 1.650 & 2.15 & 0.041 & 0.026 & 0.007 \\
\hline KA11 & 0.73 & 0.33 & 0.014 & 0.049 & 0.027 & 1.359 & 1.68 & 0.014 & 0.009 & 0.008 \\
\hline KA12 & 1.59 & 0.71 & 0.050 & 0.335 & 0.071 & 1.830 & 5.25 & 0.017 & 0.055 & 0.007 \\
\hline KA12 & 1.99 & 0.55 & 0.126 & 0.429 & 0.111 & 1.920 & 3.58 & 0.022 & 0.038 & 0.015 \\
\hline KA12 & 1.00 & 0.46 & 0.050 & 0.287 & 0.137 & 1.045 & 4.19 & 0.022 & 0.104 & 0.010 \\
\hline KA13 & 0.25 & 0.24 & 0.014 & 0.055 & 0.023 & 0.307 & 1.27 & 0.008 & 0.012 & 0.007 \\
\hline KA13 & 0.51 & 0.23 & 0.055 & 0.068 & 0.018 & 0.599 & 0.76 & 0.012 & 0.022 & 0.009 \\
\hline KA13 & 0.89 & 0.36 & 0.038 & 0.121 & 0.015 & 0.791 & 3.27 & 0.013 & 0.026 & 0.010 \\
\hline KA14 & 1.13 & 0.79 & 0.031 & 0.106 & 0.020 & 2.010 & 8.15 & 0.028 & 0.014 & 0.011 \\
\hline KA14 & 1.45 & 1.23 & 0.093 & 0.259 & 0.031 & 2.680 & 13.96 & 0.046 & 0.036 & 0.013 \\
\hline KA14 & 1.26 & 1.14 & 0.069 & 0.181 & 0.022 & 3.060 & 9.35 & 0.030 & 0.050 & 0.010 \\
\hline KA15 & 3.37 & 2.30 & 0.023 & 0.391 & 0.016 & 5.460 & 5.93 & 0.024 & 0.032 & 0.009 \\
\hline KA15 & 1.32 & 0.69 & 0.025 & 0.202 & 0.018 & 2.180 & 2.34 & 0.044 & 0.064 & 0.006 \\
\hline KA15 & 4.15 & 3.24 & 0.046 & 0.286 & 0.013 & 6.520 & 12.56 & 0.034 & 0.896 & 0.011 \\
\hline
\end{tabular}


$\begin{array}{lllllllllll}\text { Sample No. } & \text { Nd146 } & \text { Sm147 } & \text { Eu153 } & \text { Gd157 } & \text { Dy163 } & \text { Er166 } & \text { Yb172 } & \text { Pb208 } & \text { Th232 } & \text { U238 }\end{array}$

\begin{tabular}{|c|c|c|c|c|c|c|c|c|c|c|}
\hline KA1 & 0.061 & 0.073 & 0.014 & 0.049 & 0.027 & 0.027 & 0.047 & 0.03 & 0.011 & 3.620 \\
\hline KA1 & 0.049 & 0.029 & 0.013 & 0.048 & 0.024 & 0.026 & 0.032 & 0.02 & 0.012 & 4.550 \\
\hline KA1 & 0.040 & 0.028 & 0.015 & 0.033 & 0.029 & 0.021 & 0.025 & 0.04 & 0.010 & 2.430 \\
\hline KA2 & 0.058 & 0.044 & 0.012 & 0.051 & 0.032 & 0.034 & 0.040 & 0.03 & 0.011 & 5.130 \\
\hline KA2 & 0.050 & 0.043 & 0.016 & 0.056 & 0.043 & 0.026 & 0.038 & 0.08 & 0.012 & 4.500 \\
\hline KA2 & 0.058 & 0.062 & 0.018 & 0.041 & 0.026 & 0.024 & 0.020 & 0.03 & 0.015 & 3.060 \\
\hline KA3 & 0.047 & 0.067 & 0.018 & 0.053 & 0.023 & 0.019 & 0.026 & 0.14 & 0.010 & 6.240 \\
\hline KA3 & 0.094 & 0.051 & 0.012 & 0.054 & 0.071 & 0.077 & 0.040 & 0.12 & 0.010 & 6.390 \\
\hline KA3 & 0.294 & 0.072 & 0.048 & 0.235 & 0.334 & 0.374 & 0.434 & 2.87 & 0.015 & 7.510 \\
\hline KA4 & 0.035 & 0.048 & 0.011 & 0.045 & 0.024 & 0.026 & 0.022 & 0.11 & 0.006 & 4.400 \\
\hline KA4 & 0.059 & 0.054 & 0.005 & 0.042 & 0.023 & 0.020 & 0.028 & 0.11 & 0.007 & 5.290 \\
\hline KA4 & 0.265 & 0.060 & 0.010 & 0.047 & 0.032 & 0.032 & 0.029 & 0.84 & 0.193 & 5.360 \\
\hline KA5 & 0.060 & 0.045 & 0.015 & 0.074 & 0.033 & 0.038 & 0.041 & 0.47 & 0.005 & 0.393 \\
\hline KA5 & 0.635 & 0.261 & 0.073 & 0.494 & 0.804 & 0.687 & 0.858 & 2.99 & 0.023 & 1.097 \\
\hline KA5 & 0.311 & 0.163 & 0.051 & 0.304 & 0.609 & 0.499 & 0.733 & 1.33 & 0.015 & 0.701 \\
\hline KA6 & 0.118 & 0.141 & 0.033 & 0.103 & 0.051 & 0.048 & 0.040 & 0.06 & 0.024 & 11.330 \\
\hline KA6 & 0.051 & 0.105 & 0.011 & 0.100 & 0.051 & 0.043 & 0.027 & 0.04 & 2.020 & 7.950 \\
\hline KA6 & 0.065 & 0.064 & 0.017 & 0.104 & 0.070 & 0.035 & 0.086 & 0.07 & 1.750 & 5.650 \\
\hline KA7 & 0.065 & 0.070 & 0.022 & 0.087 & 0.036 & 0.035 & 0.063 & 0.04 & 0.012 & 3.850 \\
\hline KA7 & 0.052 & 0.051 & 0.009 & 0.076 & 0.042 & 0.039 & 0.051 & 0.04 & 0.014 & 4.250 \\
\hline KA7 & 0.053 & 0.078 & 0.018 & 0.081 & 0.036 & 0.044 & 0.039 & 0.03 & 0.012 & 4.550 \\
\hline KA8 & 0.038 & 0.056 & 0.009 & 0.037 & 0.019 & 0.014 & 0.025 & 0.13 & 0.006 & 13.110 \\
\hline KA8 & 0.046 & 0.048 & 0.015 & 0.059 & 0.028 & 0.017 & 0.035 & 0.08 & 0.010 & 8.800 \\
\hline KA8 & 0.022 & 0.036 & 0.012 & 0.049 & 0.016 & 0.020 & 0.037 & 0.06 & 0.009 & 10.110 \\
\hline KA9 & 0.060 & 0.064 & 0.017 & 0.053 & 0.038 & 0.028 & 0.021 & 0.03 & 0.010 & 1.227 \\
\hline KA9 & 0.065 & 0.055 & 0.020 & 0.052 & 0.037 & 0.024 & 0.029 & 0.02 & 0.013 & 1.560 \\
\hline KA9 & 0.037 & 0.054 & 0.014 & 0.042 & 0.037 & 0.024 & 0.035 & 0.22 & 0.013 & 1.330 \\
\hline KA10 & 0.078 & 0.066 & 0.009 & 0.040 & 0.035 & 0.032 & 0.050 & 0.61 & 0.061 & 2.160 \\
\hline KA10 & 0.066 & 0.052 & 0.011 & 0.040 & 0.021 & 0.026 & 0.038 & 0.13 & 0.036 & 1.750 \\
\hline KA10 & 0.134 & 0.051 & 0.021 & 0.070 & 0.043 & 0.030 & 0.051 & 0.14 & 0.015 & 3.060 \\
\hline KA11 & 0.051 & 0.039 & 0.007 & 0.037 & 0.036 & 0.017 & 0.035 & 0.09 & 0.008 & 2.510 \\
\hline KA11 & 0.047 & 0.040 & 0.017 & 0.065 & 0.023 & 0.025 & 0.036 & 0.06 & 0.012 & 2.800 \\
\hline KA11 & 0.058 & 0.031 & 0.018 & 0.066 & 0.026 & 0.024 & 0.023 & 0.07 & 0.013 & 2.260 \\
\hline KA12 & 0.051 & 0.050 & 0.016 & 0.082 & 0.055 & 0.031 & 0.032 & 0.12 & 0.014 & 2.040 \\
\hline KA12 & 0.040 & 0.067 & 0.015 & 0.064 & 0.080 & 0.051 & 0.037 & 0.15 & 0.399 & 1.970 \\
\hline KA12 & 0.068 & 0.041 & 0.015 & 0.094 & 0.024 & 0.031 & 0.045 & 0.16 & 0.019 & 1.980 \\
\hline KA13 & 0.057 & 0.083 & 0.009 & 0.072 & 0.035 & 0.021 & 0.061 & 0.05 & 0.014 & 0.496 \\
\hline KA13 & 0.047 & 0.066 & 0.018 & 0.094 & 0.055 & 0.027 & 0.067 & 0.04 & 0.013 & 0.490 \\
\hline KA13 & 0.059 & 0.078 & 0.024 & 0.047 & 0.036 & 0.031 & 0.063 & 0.04 & 0.015 & 0.492 \\
\hline KA14 & 0.059 & 0.065 & 0.019 & 0.055 & 0.030 & 0.021 & 0.040 & 0.06 & 0.013 & 3.370 \\
\hline KA14 & 0.062 & 0.048 & 0.013 & 0.053 & 0.033 & 0.037 & 0.031 & 0.12 & 0.009 & 3.490 \\
\hline KA14 & 0.041 & 0.070 & 0.020 & 0.078 & 0.035 & 0.036 & 0.040 & 0.13 & 0.011 & 3.710 \\
\hline KA15 & 0.047 & 0.064 & 0.021 & 0.053 & 0.047 & 0.032 & 0.065 & 0.06 & 0.013 & 1.930 \\
\hline KA15 & 0.055 & 0.077 & 0.015 & 0.048 & 0.042 & 0.023 & 0.046 & 0.15 & 0.013 & 2.520 \\
\hline KA15 & 0.035 & 0.059 & 0.019 & 0.055 & 0.018 & 0.030 & 0.038 & 0.22 & 0.009 & 1.390 \\
\hline
\end{tabular}




\begin{tabular}{|c|c|c|c|c|c|c|c|c|c|c|}
\hline Sample No. & $\mathrm{Li} 7$ & Be9 & B11 & $\operatorname{Mg} 24$ & $\mathrm{Al} 27$ & P31 & K39 & $\mathrm{Ca} 43$ & Ti49 & V51 \\
\hline KR1 & 0.2 & 0.13 & 6.2 & 9.2 & 62.2 & 27.48 & 31.4 & 100.9 & 0.74 & 0.30 \\
\hline KR1 & 0.3 & 0.21 & 6.3 & 13.6 & 54.9 & 11.63 & 27.9 & 114.3 & 0.89 & 0.34 \\
\hline KR1 & 0.2 & 0.12 & 6.1 & 9.2 & 58.3 & 19.49 & 21.8 & 187.3 & 0.82 & 0.23 \\
\hline KR2 & 0.2 & 0.19 & 5.9 & 13.8 & 195.7 & 17.89 & 42.5 & 105.6 & 0.75 & 0.98 \\
\hline KR2 & 0.2 & 0.10 & 5.7 & 11.5 & 167.6 & 10.40 & 37.0 & 119.3 & 0.80 & 0.94 \\
\hline KR2 & 0.3 & 0.08 & 4.7 & 7.3 & 58.1 & 10.83 & 23.8 & 96.2 & 0.73 & 1.48 \\
\hline KR3 & 0.3 & 0.17 & 4.8 & 5.8 & 24.4 & 15.60 & 25.5 & 107.6 & 1.00 & 1.09 \\
\hline KR3 & 0.3 & 0.16 & 4.5 & 5.9 & 31.6 & 14.02 & 22.8 & 120.7 & 0.75 & 0.94 \\
\hline KR3 & 0.3 & 0.15 & 6.0 & 7.9 & 36.6 & 11.95 & 28.8 & 106.1 & 1.06 & 1.00 \\
\hline KR4 & 0.6 & 0.30 & 2.4 & 8.3 & 4.4 & 22.34 & 10.6 & 225.7 & 1.24 & 2.08 \\
\hline KR4 & 0.4 & 0.31 & 5.2 & 6.2 & 11.3 & 16.76 & 13.6 & 136.8 & 1.24 & 3.27 \\
\hline KR4 & 0.3 & 0.17 & 2.2 & 11.6 & 4.4 & 26.77 & 7.4 & 616.5 & 0.68 & 22.86 \\
\hline KR5 & 0.4 & 0.11 & 5.2 & 8.1 & 99.6 & 14.42 & 72.1 & 128.6 & 1.08 & 1.41 \\
\hline KR5 & 0.5 & 0.20 & 4.1 & 8.1 & 65.5 & 17.72 & 56.8 & 173.3 & 0.75 & 1.00 \\
\hline KR5 & 0.3 & 0.18 & 4.8 & 7.0 & 76.2 & 18.30 & 48.8 & 108.7 & 1.06 & 0.78 \\
\hline KR6 & 0.4 & 0.30 & 5.2 & 8.3 & 13.2 & 17.31 & 13.3 & 148.0 & 1.19 & 0.40 \\
\hline KR6 & 3.6 & n.d. & 4.5 & 67.1 & 180.1 & 13.17 & 43.4 & 203.2 & 3.19 & 0.58 \\
\hline KR6 & 0.6 & 0.21 & 5.9 & 11.3 & 81.8 & 23.63 & 40.0 & 204.7 & 1.14 & 0.47 \\
\hline KR7 & 0.4 & 0.21 & 35.2 & 26.6 & 46.1 & 17.01 & 20.5 & 156.5 & 1.14 & 0.86 \\
\hline KR7 & 0.7 & 0.34 & 45.9 & 24.9 & 45.1 & 26.73 & 17.6 & 242.3 & 1.50 & 1.16 \\
\hline KR7 & 0.7 & 0.23 & 42.5 & 9.7 & 29.3 & 28.34 & 35.7 & 244.6 & 1.68 & 0.97 \\
\hline KR8 & 4.6 & 3.27 & 12.8 & 115.3 & 24.4 & 195.20 & 71.0 & 1689.9 & 13.93 & 4.11 \\
\hline KR8 & 0.7 & 0.37 & 5.5 & 18.5 & 33.9 & 28.56 & 28.2 & 260.4 & 3.36 & 6.72 \\
\hline KR8 & 0.5 & 0.32 & 2.3 & 38.2 & 10.6 & 19.33 & 13.9 & 178.8 & 0.97 & 3.43 \\
\hline KR9 & 0.6 & 0.19 & 4.7 & 6.3 & 37.3 & 26.46 & 44.1 & 260.6 & 0.59 & 0.48 \\
\hline KR9 & 0.3 & 0.12 & 4.3 & 3.7 & 19.9 & 12.76 & 46.9 & 115.5 & 0.80 & 0.43 \\
\hline KR9 & 0.8 & 0.17 & 4.4 & 5.7 & 31.1 & 17.04 & 57.6 & 230.9 & 1.02 & 0.61 \\
\hline KR10 & 0.4 & 0.24 & 9.7 & 7.3 & 16.4 & 38.00 & 31.6 & 127.6 & 1.94 & 0.45 \\
\hline KR10 & 0.7 & 0.21 & 14.5 & 9.2 & 107.4 & 175.05 & 63.2 & 226.7 & 1.89 & 0.14 \\
\hline KR10 & 1.3 & 0.36 & 12.5 & 24.7 & 160.3 & 19.16 & 87.9 & 168.3 & 2.77 & 0.19 \\
\hline KR11 & 0.3 & 0.12 & 3.3 & 5.3 & 27.2 & 11.43 & 19.4 & 96.6 & 2.10 & 0.43 \\
\hline KR11 & 0.5 & 0.33 & 3.6 & 5.6 & 18.5 & 24.57 & 15.9 & 179.2 & 1.22 & 0.54 \\
\hline KR11 & 0.4 & 0.21 & 3.6 & 4.8 & 18.9 & 13.61 & 19.0 & 114.4 & 0.99 & 0.61 \\
\hline KR12 & 0.4 & 0.29 & 5.9 & 9.5 & 29.3 & 22.80 & 22.0 & 220.4 & 0.88 & 0.73 \\
\hline KR12 & 0.3 & 0.15 & 6.8 & 5.4 & 55.3 & 11.30 & 23.4 & 102.1 & 1.37 & 0.65 \\
\hline KR12 & 0.5 & 0.14 & 4.5 & 4.2 & 15.7 & 21.78 & 21.0 & 151.5 & 1.05 & 0.60 \\
\hline KR13 & 0.9 & 1.28 & 10.6 & 120.8 & 34.5 & 33.41 & 60.5 & 285.7 & 2.97 & 0.91 \\
\hline KR13 & 0.9 & 0.45 & 8.3 & 75.7 & 23.4 & 31.54 & 43.2 & 271.7 & 3.76 & 0.73 \\
\hline KR13 & n.d. & 1.27 & 7.5 & 27.2 & 84.2 & 34.24 & 92.9 & n.d. & 338.44 & 1.46 \\
\hline KR14 & 0.7 & 0.16 & 7.4 & 10.1 & 240.3 & 13.92 & 53.8 & 202.8 & 1.43 & 0.98 \\
\hline KR14 & 1.0 & 0.10 & 9.0 & 10.4 & 231.6 & 10.57 & 52.8 & 186.9 & 0.77 & 0.88 \\
\hline KR14 & 0.6 & 0.13 & 7.4 & 8.4 & 189.1 & 16.90 & 33.9 & 178.7 & 0.70 & 1.05 \\
\hline KR15 & 0.3 & 0.28 & 3.9 & 5.5 & 16.3 & 19.94 & 32.3 & 103.2 & 0.80 & 1.04 \\
\hline KR15 & 0.4 & 0.26 & 4.3 & 4.6 & 7.4 & 16.55 & 20.4 & 176.7 & 0.95 & 1.01 \\
\hline KR15 & 0.4 & 0.32 & 3.7 & 4.9 & 18.4 & 15.41 & 28.1 & 137.1 & 1.03 & 1.16 \\
\hline
\end{tabular}




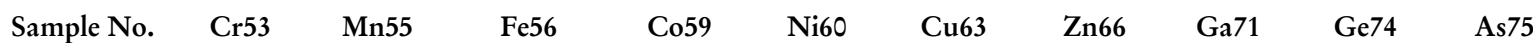

\begin{tabular}{|c|c|c|c|c|c|c|c|c|c|c|}
\hline KR1 & 1.38 & 0.5 & 40 & 0.03 & 0.71 & 0.22 & 0.78 & 0.071 & 1.150 & 1.8 \\
\hline KR1 & 1.61 & 0.3 & 30 & 0.05 & 0.49 & 0.24 & 0.79 & 0.064 & 1.760 & 2.4 \\
\hline KR1 & 1.58 & 0.3 & 32 & 0.02 & 0.70 & 0.22 & 0.64 & 0.041 & 1.000 & 1.6 \\
\hline KR2 & 1.40 & 0.7 & 15.2 & 0.03 & 0.13 & 0.23 & 0.28 & 0.064 & 1.930 & 0.4 \\
\hline KR2 & 1.46 & 0.5 & 14.4 & 0.03 & 0.28 & 0.22 & 0.35 & 0.068 & 2.280 & 0.3 \\
\hline KR2 & 1.46 & 0.3 & 8.7 & 0.02 & 1.02 & 0.23 & 0.49 & 0.047 & 1.910 & 0.4 \\
\hline KR3 & 1.75 & 0.3 & 15 & 0.04 & 0.52 & 0.28 & 0.33 & 0.069 & 1.730 & 0.4 \\
\hline KR3 & 1.59 & 0.3 & 14 & 0.03 & 0.43 & 0.24 & 0.33 & 0.057 & 1.960 & 0.4 \\
\hline KR3 & 1.63 & 0.3 & 23 & 0.04 & 0.37 & 0.24 & 0.27 & 0.055 & 2.010 & 0.4 \\
\hline KR4 & 2.91 & 0.6 & 44.3 & 0.06 & 0.36 & 0.52 & 0.85 & 0.091 & 2.050 & 0.6 \\
\hline KR4 & 2.21 & 0.5 & 93.0 & 0.05 & 0.57 & 0.42 & 0.52 & 0.076 & 2.280 & 0.7 \\
\hline KR4 & 1.42 & 2.3 & 471.5 & 0.08 & 0.60 & 0.78 & 0.33 & 0.043 & 1.560 & 7.5 \\
\hline KR5 & 1.82 & 0.4 & 98 & 0.03 & 0.32 & 0.33 & 0.50 & 0.071 & 1.740 & 0.5 \\
\hline KR5 & 1.76 & 0.4 & 109 & 0.03 & 0.31 & 0.30 & 1.09 & 0.047 & 2.020 & 0.5 \\
\hline KR5 & 1.65 & 0.3 & 84 & 0.05 & 0.42 & 0.29 & 0.35 & 0.060 & 2.400 & 0.4 \\
\hline KR6 & 2.27 & 0.5 & 75.6 & 0.05 & 0.51 & 0.53 & 0.66 & 0.594 & 0.640 & 1.7 \\
\hline KR6 & n.d. & 0.1 & 81.0 & n.d. & 1.35 & 3.00 & 4.93 & 0.650 & n.d. & n.d. \\
\hline KR6 & 3.24 & 0.7 & 93.9 & 0.09 & 0.69 & 0.57 & 0.76 & 0.617 & 0.770 & 3.5 \\
\hline KR7 & 2.28 & 0.5 & 32 & 0.04 & 0.48 & 0.50 & 0.62 & 0.980 & 2.140 & 1.0 \\
\hline KR7 & 3.50 & 0.8 & 30 & 0.11 & 0.72 & 0.61 & 0.95 & 1.030 & 2.160 & 1.3 \\
\hline KR7 & 3.72 & 0.8 & 40 & 0.08 & 0.33 & 0.62 & 1.05 & 0.760 & 2.820 & 1.0 \\
\hline KR8 & 27.46 & 5.6 & 104.6 & 0.77 & 2.48 & 4.43 & 6.71 & 1.400 & 4.850 & 7.0 \\
\hline KR8 & 3.79 & 0.8 & 100.3 & 0.10 & 0.69 & 0.68 & 0.87 & 0.171 & 1.350 & 3.2 \\
\hline KR8 & 2.75 & 0.5 & 62.1 & 0.05 & 0.93 & 0.92 & 0.61 & 0.099 & 2.430 & 0.6 \\
\hline KR9 & 1.72 & 0.4 & 116 & 0.04 & 0.18 & 0.28 & 0.39 & 0.064 & 1.570 & 1.9 \\
\hline KR9 & 1.63 & 0.4 & 115 & 0.03 & 0.18 & 0.29 & 0.35 & 0.072 & 2.110 & 1.3 \\
\hline KR9 & 1.82 & 0.4 & 172 & 0.04 & 0.19 & 0.33 & 0.37 & 0.057 & 2.480 & 2.0 \\
\hline KR10 & 1.73 & 0.4 & 79.6 & 0.04 & 0.48 & 0.67 & 0.43 & 0.075 & 8.110 & 5.1 \\
\hline KR10 & 3.38 & 0.7 & 22.8 & 0.07 & 0.64 & 0.62 & 1.02 & 0.130 & 15.360 & 12.8 \\
\hline KR10 & 2.51 & 0.5 & 21.0 & 0.07 & 0.44 & 0.43 & 0.46 & 0.474 & 13.110 & 9.8 \\
\hline KR11 & 1.45 & 0.3 & 86 & 0.05 & 0.20 & 0.29 & 0.66 & 0.070 & 1.930 & 1.3 \\
\hline KR11 & 2.42 & 0.5 & 88 & 0.06 & 0.29 & 0.48 & 0.55 & 0.126 & 2.830 & 0.8 \\
\hline KR11 & 1.69 & 0.4 & 67 & 0.04 & 0.21 & 0.36 & 0.50 & 0.100 & 2.500 & 0.5 \\
\hline KR12 & 1.70 & 0.6 & 22.9 & 0.06 & 0.38 & 0.32 & 0.31 & 0.077 & 1.280 & 0.8 \\
\hline KR12 & 1.33 & 0.3 & 26.4 & 0.03 & 0.20 & 0.28 & 0.30 & 0.073 & 1.750 & 0.4 \\
\hline KR12 & 2.28 & 0.5 & 48.7 & 0.06 & 0.31 & 0.44 & 0.59 & 0.091 & 1.210 & 0.8 \\
\hline KR13 & 4.14 & 0.9 & 118 & 2.70 & 1.11 & 0.76 & 6.64 & 0.178 & 3.350 & 1.7 \\
\hline KR13 & 3.82 & 0.9 & 57 & 0.10 & 0.68 & 1.02 & 4.60 & 0.159 & 2.050 & 1.2 \\
\hline KR13 & n.d. & 0.7 & 103 & 0.03 & 0.52 & 0.69 & 55.76 & 0.045 & 2.680 & 2.3 \\
\hline KR14 & 1.18 & 0.3 & 46.4 & 0.03 & 0.12 & 0.24 & 0.18 & 0.056 & 0.730 & 0.3 \\
\hline KR14 & 1.07 & 0.3 & 44.5 & 0.03 & 0.12 & 0.24 & 0.29 & 0.042 & 0.970 & 0.3 \\
\hline KR14 & 1.18 & 0.3 & 11.1 & 0.04 & 0.18 & 0.24 & 0.35 & 0.057 & 0.550 & 0.2 \\
\hline KR15 & 1.61 & 0.4 & 78 & 0.05 & 0.63 & 0.41 & 0.33 & 0.072 & 1.640 & 0.7 \\
\hline KR15 & 1.69 & 0.4 & 69 & 0.06 & 0.76 & 0.44 & 0.31 & 0.147 & 2.070 & 1.1 \\
\hline KR15 & 1.80 & 0.4 & 103 & 0.04 & 0.49 & 0.98 & 0.63 & 0.141 & 1.840 & 0.8 \\
\hline
\end{tabular}




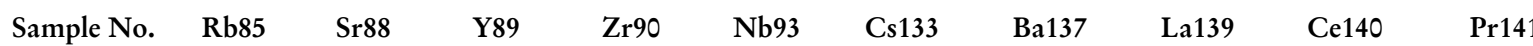

\begin{tabular}{|c|c|c|c|c|c|c|c|c|c|c|}
\hline KR1 & 0.18 & 0.47 & 0.464 & 0.054 & 0.628 & 0.096 & 0.90 & 0.239 & 0.155 & 0.041 \\
\hline KR1 & 0.22 & 0.58 & 0.111 & 0.022 & 0.686 & 0.114 & 1.76 & 0.070 & 0.038 & 0.009 \\
\hline KR1 & 0.19 & 0.50 & 0.271 & 0.021 & 0.641 & 0.099 & 1.18 & 0.142 & 0.107 & 0.025 \\
\hline KR2 & 0.20 & 0.40 & 0.015 & 0.199 & 0.156 & 0.097 & 0.83 & 0.008 & 0.008 & 0.004 \\
\hline KR2 & 0.19 & 0.33 & 0.015 & 0.217 & 0.097 & 0.104 & 0.72 & 0.007 & 0.008 & 0.007 \\
\hline KR2 & 0.19 & 0.22 & 0.011 & 0.017 & 0.044 & 0.105 & 0.47 & 0.005 & 0.011 & 0.009 \\
\hline KR3 & 0.21 & 0.17 & 0.025 & 0.024 & 0.045 & 0.122 & 0.19 & 0.013 & 0.016 & 0.011 \\
\hline KR3 & 0.20 & 0.18 & 0.015 & 0.019 & 0.043 & 0.109 & 0.18 & 0.012 & 0.014 & 0.007 \\
\hline KR3 & 0.22 & 0.22 & 0.010 & 0.019 & 0.061 & 0.118 & 0.22 & 0.013 & 0.017 & 0.012 \\
\hline KR4 & 0.42 & 0.27 & 0.050 & 0.055 & 0.049 & 0.235 & 0.43 & 0.013 & 0.019 & 0.023 \\
\hline KR4 & 0.32 & 0.27 & 0.021 & 0.036 & 0.024 & 0.170 & 0.65 & 0.011 & 0.018 & 0.014 \\
\hline KR4 & 0.21 & 0.86 & 0.116 & 0.013 & 0.022 & 0.108 & 0.85 & 0.077 & 0.130 & 0.038 \\
\hline KR5 & 0.28 & 0.34 & 0.043 & 0.439 & 0.405 & 0.244 & 0.71 & 0.018 & 0.034 & 0.010 \\
\hline KR5 & 0.26 & 0.35 & 0.023 & 0.323 & 0.448 & 0.141 & 0.58 & 0.014 & 0.015 & 0.014 \\
\hline KR5 & 0.23 & 0.38 & 0.215 & 0.324 & 0.512 & 0.126 & 1.08 & 0.086 & 0.181 & 0.021 \\
\hline KR6 & 0.34 & 0.20 & 0.022 & 0.087 & 0.138 & 0.181 & 0.89 & 0.018 & 0.017 & 0.009 \\
\hline KR6 & n.d. & 0.05 & 0.161 & 0.080 & 0.132 & n.d. & 1.05 & n.d. & n.d. & n.d. \\
\hline KR6 & 0.43 & 0.28 & 0.025 & 0.158 & 0.149 & 0.255 & 0.83 & 0.024 & 0.019 & 0.019 \\
\hline KR7 & 0.31 & 0.23 & 0.029 & 0.619 & 0.091 & 0.171 & 0.43 & 0.017 & 0.018 & 0.011 \\
\hline KR7 & 0.51 & 0.28 & 0.023 & 0.803 & 0.151 & 0.280 & 0.29 & 0.018 & 0.022 & 0.021 \\
\hline KR7 & 0.53 & 0.28 & 0.018 & 0.471 & 0.077 & 0.290 & 0.36 & 0.019 & 0.019 & 0.011 \\
\hline KR8 & 3.56 & 0.42 & 0.209 & 4.040 & 1.940 & 1.980 & 1.37 & 0.158 & 0.093 & 0.206 \\
\hline KR8 & 0.52 & 0.37 & 0.025 & 2.240 & 0.776 & 0.280 & 0.79 & 0.019 & 0.027 & 0.011 \\
\hline KR8 & 0.46 & 0.17 & 0.021 & 1.113 & 0.477 & 0.202 & 0.55 & 0.016 & 0.014 & 0.015 \\
\hline KR9 & 0.29 & 3.96 & 0.206 & 0.038 & 0.046 & 0.117 & 156.61 & 0.279 & 0.493 & 0.068 \\
\hline KR9 & 0.34 & 0.73 & 0.198 & 0.028 & 0.027 & 0.192 & 35.99 & 0.203 & 0.489 & 0.055 \\
\hline KR9 & 0.54 & 0.99 & 0.226 & 0.022 & 0.018 & 0.134 & 27.20 & 0.308 & 0.720 & 0.057 \\
\hline KR10 & 0.26 & 0.57 & 0.187 & 1.014 & 1.511 & 0.146 & 0.98 & 0.132 & 0.034 & 0.024 \\
\hline KR10 & 0.52 & 0.56 & 0.038 & 1.091 & 0.944 & 0.290 & 0.47 & 0.018 & 0.026 & 0.014 \\
\hline KR10 & 0.39 & 0.70 & 0.019 & 4.070 & 1.435 & 0.565 & 0.84 & 0.024 & 0.016 & 0.019 \\
\hline KR11 & 0.22 & 0.28 & 0.015 & 0.078 & 0.077 & 0.126 & 0.30 & 0.014 & 0.013 & 0.003 \\
\hline KR11 & 0.36 & 0.25 & 0.017 & 0.089 & 0.052 & 0.198 & 0.29 & 0.016 & 0.023 & 0.013 \\
\hline KR11 & 0.27 & 0.20 & 0.022 & 0.034 & 0.055 & 0.147 & 0.38 & 0.011 & 0.012 & 0.014 \\
\hline KR12 & 0.27 & 0.38 & 0.035 & 0.108 & 0.018 & 0.148 & 3.17 & 0.019 & 0.015 & 0.010 \\
\hline KR12 & 0.22 & 0.29 & 0.037 & 0.117 & 0.049 & 0.121 & 0.67 & 0.026 & 0.013 & 0.009 \\
\hline KR12 & 0.35 & 0.17 & 0.026 & 0.087 & 0.050 & 0.192 & 0.57 & 0.020 & 0.020 & 0.013 \\
\hline KR13 & 0.78 & 0.30 & 0.084 & 0.033 & 0.037 & 0.340 & 0.73 & 0.080 & 0.321 & 0.023 \\
\hline KR13 & 0.63 & 8.63 & 0.429 & 0.100 & 0.038 & 0.340 & 1.09 & 0.476 & 0.827 & 0.133 \\
\hline KR13 & n.d. & 0.28 & 0.014 & n.d. & 0.333 & 0.030 & 0.55 & 0.032 & 0.231 & 0.009 \\
\hline KR14 & 0.19 & 0.70 & 0.022 & 0.108 & 0.123 & 0.102 & 2.38 & 0.011 & 0.010 & 0.007 \\
\hline KR14 & 0.19 & 0.69 & 0.011 & 0.145 & 0.092 & 0.100 & 2.07 & 0.004 & 0.008 & 0.005 \\
\hline KR14 & 0.18 & 0.64 & 0.020 & 0.034 & 0.066 & 0.107 & 1.95 & 0.009 & 0.009 & 0.006 \\
\hline KR15 & 0.26 & 0.35 & 0.034 & 0.067 & 0.021 & 0.146 & 1.09 & 0.018 & 0.058 & 0.009 \\
\hline KR15 & 0.29 & 0.20 & 0.016 & 0.047 & 0.028 & 0.148 & 0.24 & 0.018 & 0.029 & 0.012 \\
\hline KR15 & 0.31 & 0.25 & 0.026 & 0.099 & 0.022 & 0.171 & 0.31 & 0.015 & 0.032 & 0.013 \\
\hline
\end{tabular}


$\begin{array}{lllllllllll}\text { Sample No. } & \text { Nd146 } & \text { Sm147 } & \text { Eu153 } & \text { Gd157 } & \text { Dy163 } & \text { Er166 } & \text { Yb172 } & \text { Pb208 } & \text { Th232 } & \text { U238 }\end{array}$

\begin{tabular}{|c|c|c|c|c|c|c|c|c|c|c|}
\hline KR1 & 0.148 & 0.080 & 0.010 & 0.062 & 0.044 & 0.022 & 0.040 & 1.03 & 0.010 & 4.340 \\
\hline KR1 & 0.066 & 0.070 & 0.011 & 0.048 & 0.034 & 0.018 & 0.049 & 0.68 & 2.270 & 3.580 \\
\hline KR1 & 0.134 & 0.058 & 0.021 & 0.026 & 0.036 & 0.021 & 0.031 & 0.81 & 0.010 & 4.020 \\
\hline KR2 & 0.039 & 0.081 & 0.014 & 0.051 & 0.031 & 0.017 & 0.038 & 0.03 & 0.011 & 1.482 \\
\hline KR2 & 0.049 & 0.019 & 0.010 & 0.050 & 0.035 & 0.026 & 0.034 & 0.03 & 0.010 & 1.422 \\
\hline KR2 & 0.031 & 0.062 & 0.013 & 0.037 & 0.043 & 0.024 & 0.030 & 0.05 & 0.013 & 1.235 \\
\hline KR3 & 0.060 & 0.045 & 0.017 & 0.030 & 0.032 & 0.024 & 0.045 & 0.05 & 0.011 & 1.519 \\
\hline KR3 & 0.043 & 0.030 & 0.021 & 0.040 & 0.035 & 0.023 & 0.030 & 0.03 & 0.009 & 1.401 \\
\hline KR3 & 0.058 & 0.070 & 0.016 & 0.051 & 0.026 & 0.024 & 0.039 & 0.03 & 0.007 & 1.650 \\
\hline KR4 & 0.117 & 0.154 & 0.023 & 0.060 & 0.090 & 0.039 & 0.056 & 0.07 & 0.021 & 1.370 \\
\hline KR4 & 0.039 & 0.081 & 0.030 & 0.109 & 0.067 & 0.036 & 0.052 & 0.27 & 0.076 & 1.900 \\
\hline KR4 & 0.096 & 0.080 & 0.021 & 0.049 & 0.045 & 0.026 & 0.043 & 1.90 & 3.430 & 1.052 \\
\hline KR5 & 0.074 & 0.040 & 0.023 & 0.053 & 0.057 & 0.025 & 0.044 & 0.10 & 0.012 & 1.210 \\
\hline KR5 & 0.062 & 0.065 & 0.024 & 0.070 & 0.031 & 0.033 & 0.047 & 0.05 & 0.011 & 1.094 \\
\hline KR5 & 0.164 & 0.075 & 0.015 & 0.055 & 0.058 & 0.063 & 0.048 & 0.07 & 0.067 & 1.017 \\
\hline KR6 & 0.057 & 0.084 & 0.038 & 0.079 & 0.064 & 0.021 & 0.031 & 0.05 & 5.060 & 2.110 \\
\hline KR6 & n.d. & 0.180 & 0.047 & n.d. & 0.110 & 0.080 & 0.230 & n.d. & 14.920 & 4.890 \\
\hline KR6 & 0.076 & 0.128 & 0.034 & 0.121 & 0.065 & 0.056 & 0.058 & 0.11 & 14.860 & 2.440 \\
\hline KR7 & 0.065 & 0.064 & 0.006 & 0.061 & 0.070 & 0.040 & 0.076 & 0.05 & 8.560 & 0.981 \\
\hline KR7 & 0.086 & 0.073 & 0.019 & 0.183 & 0.096 & 0.046 & 0.080 & 0.07 & 12.920 & 1.115 \\
\hline KR7 & 0.130 & 0.079 & 0.041 & 0.105 & 0.138 & 0.060 & 0.111 & 0.08 & 0.231 & 0.978 \\
\hline KR8 & 0.630 & 0.540 & 0.320 & 1.140 & 0.630 & 0.410 & 0.900 & 0.51 & 6.110 & 5.430 \\
\hline KR8 & 0.130 & 0.192 & 0.029 & 0.148 & 0.103 & 0.049 & 0.086 & 0.10 & 27.810 & 4.530 \\
\hline KR8 & 0.096 & 0.127 & 0.033 & 0.109 & 0.068 & 0.040 & 0.046 & 0.08 & 4.070 & 2.340 \\
\hline KR9 & 0.220 & 0.070 & 0.025 & 0.044 & 0.026 & 0.020 & 0.044 & 1.17 & 0.005 & 0.585 \\
\hline KR9 & 0.225 & 0.057 & 0.028 & 0.090 & 0.040 & 0.039 & 0.043 & 0.27 & 0.278 & 0.462 \\
\hline KR9 & 0.259 & 0.083 & 0.018 & 0.116 & 0.051 & 0.031 & 0.055 & 0.18 & 0.028 & 0.559 \\
\hline KR10 & 0.097 & 0.087 & 0.018 & 0.082 & 0.056 & 0.038 & 0.049 & 0.05 & 2.040 & 1.204 \\
\hline KR10 & 0.125 & 0.130 & 0.028 & 0.158 & 0.139 & 0.066 & 0.067 & 0.07 & 4.010 & 1.019 \\
\hline KR10 & 0.044 & 0.119 & 0.024 & 0.133 & 0.054 & 0.040 & 0.083 & 0.06 & 10.940 & 1.057 \\
\hline KR11 & 0.060 & 0.056 & 0.012 & 0.061 & 0.046 & 0.032 & 0.035 & 0.03 & 0.013 & 2.120 \\
\hline KR11 & 0.063 & 0.119 & 0.031 & 0.142 & 0.054 & 0.042 & 0.058 & 0.06 & 0.024 & 2.690 \\
\hline KR11 & 0.055 & 0.067 & 0.014 & 0.072 & 0.045 & 0.034 & 0.054 & 0.04 & 0.017 & 2.320 \\
\hline KR12 & 0.057 & 0.112 & 0.021 & 0.091 & 0.046 & 0.030 & 0.035 & 0.05 & 9.030 & 2.430 \\
\hline KR12 & 0.053 & 0.055 & 0.017 & 0.060 & 0.019 & 0.024 & 0.020 & 0.04 & 0.010 & 2.540 \\
\hline KR12 & 0.066 & 0.089 & 0.019 & 0.119 & 0.052 & 0.045 & 0.056 & 0.04 & 3.370 & 2.850 \\
\hline KR13 & 0.190 & 0.131 & 0.054 & 0.152 & 0.164 & 0.082 & 0.125 & 0.10 & 282.170 & 2.660 \\
\hline KR13 & 0.173 & 0.186 & 0.042 & 0.152 & 0.109 & 0.100 & 0.118 & 0.18 & 78.790 & 2.350 \\
\hline KR13 & 0.052 & n.d. & n.d. & n.d. & 0.061 & n.d. & 0.093 & 0.34 & 46.580 & 1.850 \\
\hline KR14 & 0.043 & 0.052 & 0.015 & 0.049 & 0.030 & 0.020 & 0.049 & 0.03 & 0.013 & 0.326 \\
\hline KR14 & 0.043 & 0.044 & 0.007 & 0.034 & 0.030 & 0.023 & 0.028 & 0.03 & 0.011 & 0.300 \\
\hline KR14 & 0.052 & 0.039 & 0.010 & 0.031 & 0.037 & 0.027 & 0.025 & 0.04 & 0.010 & 0.291 \\
\hline KR15 & 0.054 & 0.075 & 0.017 & 0.086 & 0.032 & 0.039 & 0.053 & 0.04 & 0.158 & 2.890 \\
\hline KR15 & 0.047 & 0.040 & 0.018 & 0.054 & 0.058 & 0.031 & 0.044 & 0.03 & 1.730 & 2.850 \\
\hline KR15 & 0.075 & 0.078 & 0.020 & 0.095 & 0.059 & 0.048 & 0.057 & 0.05 & 0.015 & 3.130 \\
\hline
\end{tabular}




\begin{tabular}{lcccccccccc} 
Sample No. & Li7 & Be9 & B11 & Mg24 & Al27 & P31 & K39 & Ca43 & Ti49 & V51 \\
LB1 & 5.7 & 1.17 & 120.6 & 6.3 & 163.6 & 13.73 & 134.6 & 378.5 & 0.43 & 0.94 \\
LB1 & 6.0 & 1.32 & 88.2 & 22.2 & 522.6 & 63.02 & 154.0 & 2670.9 & 3.96 & 6.11 \\
LB1 & 7.4 & 0.93 & 91.1 & 10.5 & 352.9 & 27.27 & 155.8 & 553.4 & 0.57 & 1.94 \\
\hline LB2 & 2.9 & 0.27 & 34.3 & 4.6 & 81.4 & 17.27 & 65.2 & 114.1 & 0.90 & 0.07 \\
LB2 & 1.9 & 0.23 & 32.9 & 4.0 & 62.6 & 12.17 & 47.8 & 86.9 & 2.29 & 0.08 \\
LB2 & 2.5 & 0.32 & 31.1 & 4.5 & 80.7 & 11.60 & 57.7 & 97.7 & 1.06 & 0.06 \\
LB3 & 1.5 & 0.46 & 31.6 & 5.4 & 62.7 & 33.41 & 78.5 & 63.6 & 1.04 & 2.52 \\
LB3 & 0.6 & 0.43 & 29.5 & 7.6 & 50.0 & 78.80 & 53.5 & 77.5 & 1.29 & 3.57 \\
LB3 & 0.5 & 0.67 & 30.9 & 6.6 & 31.7 & 68.89 & 41.3 & 62.4 & 1.60 & 3.29 \\
LB4 & 1.0 & 0.53 & 55.0 & 4.5 & 100.0 & 19.14 & 49.5 & 72.7 & 5.96 & 0.15 \\
LB4 & 2.5 & 0.25 & 31.9 & 5.9 & 275.4 & 21.40 & 110.6 & 71.3 & 5.65 & 0.24 \\
LB4 & 2.1 & 0.54 & 51.5 & 5.9 & 250.4 & 15.66 & 101.2 & 67.4 & 9.84 & 0.20 \\
LB5 & 1.4 & 1.36 & 35.9 & 5.4 & 135.7 & 18.18 & 88.1 & 56.9 & 4.19 & 1.07 \\
LB5 & 1.4 & 1.94 & 47.9 & 4.7 & 113.9 & 16.40 & 89.9 & 69.4 & 7.72 & 1.10 \\
LB5 & 1.2 & 1.76 & 40.2 & 6.8 & 104.1 & 44.24 & 72.3 & 75.5 & 6.28 & 6.73 \\
LB6 & 1.2 & 0.60 & 18.0 & 2.4 & 30.9 & 21.75 & 24.4 & 87.6 & 0.71 & 0.05 \\
LB6 & 1.2 & 0.20 & 16.0 & 2.7 & 31.8 & 17.76 & 21.2 & 73.6 & 1.95 & 0.28 \\
LB6 & 1.2 & 0.45 & 17.3 & 2.5 & 33.0 & 11.43 & 29.2 & 75.7 & 0.95 & 0.23 \\
LB7 & 20.1 & 0.27 & 3.8 & 15.8 & 173.9 & 19.08 & 70.6 & 272.2 & 1.33 & 0.25 \\
LB7 & 13.7 & 1.12 & 17.0 & 5.5 & 445.3 & 36.82 & 186.2 & 73.3 & 3.40 & 0.13 \\
LB7 & 10.7 & 1.19 & 19.4 & 4.1 & 340.2 & 25.60 & 144.1 & 78.8 & 2.43 & 0.09
\end{tabular}

$\begin{array}{lccccccccccc}\text { Sample No. } & \text { Cr53 } & \text { Mn55 } & \text { Fe56 } & \text { Co59 } & \text { Ni60 } & \text { Cu63 } & \text { Zn66 } & \text { Ga71 } & \text { Ge74 } & \text { As75 } \\ \text { LB1 } & 0.86 & 27.0 & 275 & 0.68 & 0.75 & 2.77 & 12.44 & 0.192 & 9.270 & 354.0 \\ \text { LB1 } & 0.74 & 149.6 & 1185 & 0.77 & 1.52 & 8.54 & 33.96 & 0.117 & 9.170 & 613.2 \\ \text { LB1 } & 0.71 & 29.5 & 1208 & 0.74 & 1.01 & 2.91 & 13.37 & 0.134 & 8.080 & 283.4 \\ \text { LB2 } & 1.50 & 0.4 & 70.9 & 0.04 & 0.39 & 0.33 & 0.45 & 0.068 & 3.730 & 49.3 & 52.3 \\ \text { LB2 } & 1.32 & 0.3 & 69.2 & 0.04 & 0.28 & 0.26 & 0.35 & 0.051 & 6.590 & 52.9 \\ \text { LB2 } & 1.25 & 0.3 & 92.7 & 0.05 & 0.28 & 0.30 & 0.37 & 0.093 & 6.360 & 5.3 \\ \text { LB3 } & 0.69 & 4.5 & 2334 & 0.46 & 0.93 & 0.76 & 0.86 & 0.025 & 6.740 & 96.2 \\ \text { LB3 } & 0.81 & 25.0 & 5217 & 1.16 & 1.62 & 2.06 & 1.54 & 0.053 & 5.240 & 168.7 \\ \text { LB3 } & 0.84 & 21.7 & 4186 & 0.91 & 1.17 & 1.77 & 1.21 & 0.038 & 5.960 & 143.6 \\ \text { LB4 } & 1.04 & 2.8 & 443.4 & 0.03 & 0.56 & 0.47 & 0.63 & 0.589 & 15.240 & 70.2 \\ \text { LB4 } & 0.92 & 3.2 & 409.4 & 0.04 & 0.68 & 0.48 & 0.67 & 0.248 & 6.550 & 33.3 \\ \text { LB4 } & 0.88 & 2.6 & 292.5 & 0.03 & 0.67 & 0.57 & 0.60 & 0.298 & 10.340 & 53.2 \\ \text { LB5 } & 0.90 & 1.9 & 1003 & 0.05 & 0.46 & 8.39 & 0.22 & 0.112 & 17.230 & 132.5 \\ \text { LB5 } & 0.90 & 2.0 & 821 & 0.06 & 0.33 & 7.86 & 0.34 & 0.060 & 23.660 & 172.5 \\ \text { LB5 } & 0.69 & 9.3 & 4286 & 0.31 & 1.01 & 41.26 & 0.88 & 0.082 & 19.580 & 178.9 \\ \text { LB6 } & 1.28 & 0.3 & 33.3 & 0.03 & 0.29 & 0.27 & 0.28 & 0.154 & 8.000 & 10.2 \\ \text { LB6 } & 1.09 & 0.4 & 179.2 & 0.03 & 0.38 & 0.22 & 0.21 & 0.103 & 7.700 & 10.0 \\ \text { LB6 } & 1.03 & 0.6 & 130.6 & 0.03 & 0.29 & 0.24 & 0.32 & 0.165 & 7.930 & 12.5 \\ \text { LB7 } & 1.39 & 807.4 & 208 & 0.17 & 0.30 & 0.60 & 0.44 & 0.428 & 1.650 & 20.3 \\ \text { LB7 } & 1.06 & 8.2 & 289 & 0.11 & 0.31 & 0.56 & 0.33 & 3.240 & 4.900 & 62.5 \\ \text { LB7 } & 1.14 & 5.7 & 129 & 0.04 & 0.37 & 0.27 & 0.44 & 3.970 & 5.310 & 67.6\end{array}$




\begin{tabular}{|c|c|c|c|c|c|c|c|c|c|c|}
\hline Sample No. & Rb85 & Sr88 & Y89 & Zr90 & $\mathrm{Nb93}$ & Cs133 & Ba137 & La139 & Ce140 & Pr141 \\
\hline LB1 & 1.80 & 2.07 & 0.383 & 0.089 & 0.011 & 2.690 & 11.35 & 0.187 & 0.054 & 0.006 \\
\hline LB1 & 1.57 & 11.32 & 3.790 & 0.423 & 0.014 & 2.540 & 49.87 & 1.606 & 0.536 & 0.042 \\
\hline LB1 & 1.68 & 3.05 & 0.956 & 0.160 & 0.011 & 2.570 & 14.45 & 0.441 & 0.222 & 0.026 \\
\hline LB2 & 0.65 & 0.22 & 0.012 & 0.108 & 0.114 & 0.340 & 0.14 & 0.017 & 0.015 & 0.011 \\
\hline LB2 & 0.41 & 0.15 & 0.010 & 0.063 & 0.083 & 0.286 & 0.21 & 0.017 & 0.019 & 0.005 \\
\hline LB2 & 0.61 & 0.17 & 0.013 & 0.114 & 0.053 & 0.448 & 0.20 & 0.012 & 0.010 & 0.010 \\
\hline LB3 & 0.78 & 0.74 & 0.108 & 0.249 & 0.118 & 0.561 & 0.74 & 0.037 & 0.070 & 0.007 \\
\hline LB3 & 0.35 & 1.75 & 0.315 & 0.283 & 0.185 & 0.294 & 2.63 & 0.089 & 0.294 & 0.047 \\
\hline LB3 & 0.27 & 1.54 & 0.270 & 0.189 & 0.172 & 0.224 & 2.09 & 0.060 & 0.226 & 0.042 \\
\hline LB4 & 0.62 & 0.66 & 0.091 & 0.907 & 0.821 & 0.670 & 4.05 & 0.014 & 0.037 & 0.006 \\
\hline LB4 & 1.54 & 0.76 & 0.107 & 2.650 & 0.649 & 1.540 & 2.42 & 0.025 & 0.025 & 0.008 \\
\hline LB4 & 1.49 & 0.89 & 0.106 & 2.270 & 0.624 & 1.351 & 3.90 & 0.020 & 0.057 & 0.011 \\
\hline LB5 & 1.11 & 0.41 & 0.062 & 2.340 & 0.203 & 2.700 & 1.48 & 0.031 & 0.048 & 0.007 \\
\hline LB5 & 0.98 & 0.41 & 0.053 & 1.127 & 0.209 & 2.540 & 2.23 & 0.044 & 0.054 & 0.010 \\
\hline LB5 & 0.82 & 0.43 & 0.205 & 3.270 & 0.253 & 1.870 & 1.78 & 0.066 & 0.159 & 0.043 \\
\hline LB6 & 0.28 & 0.95 & 0.014 & 0.271 & 0.059 & 0.120 & 36.89 & 0.010 & 0.010 & 0.006 \\
\hline LB6 & 0.19 & 0.52 & 0.134 & 0.256 & 0.046 & 0.207 & 4.69 & 0.068 & 0.210 & 0.031 \\
\hline LB6 & 0.19 & 0.31 & 0.244 & 0.260 & 0.071 & 0.106 & 2.07 & 0.046 & 0.161 & 0.027 \\
\hline LB7 & 0.22 & 5.47 & 0.207 & 0.062 & 0.009 & 0.226 & 56.42 & 1.322 & 2.490 & 0.191 \\
\hline LB7 & 1.65 & 12.07 & 0.154 & 0.197 & 0.051 & 2.960 & 161.68 & 1.940 & 4.480 & 0.264 \\
\hline LB7 & 1.67 & 4.60 & 0.073 & 0.234 & 0.077 & 2.440 & 55.75 & 0.333 & 1.073 & 0.066 \\
\hline
\end{tabular}

\begin{tabular}{|c|c|c|c|c|c|c|c|c|c|c|}
\hline Sample No. & Nd146 & Sm147 & Eu153 & Gd157 & Dy163 & Er166 & Yb172 & $\mathrm{Pb} 208$ & Th232 & U238 \\
\hline LB1 & 0.033 & 0.020 & 0.009 & 0.046 & 0.023 & 0.024 & 0.025 & 32.55 & 0.006 & 2.430 \\
\hline LB1 & 0.220 & 0.048 & 0.038 & 0.267 & 0.425 & 0.315 & 0.200 & 187.79 & 0.025 & 4.200 \\
\hline LB1 & 0.110 & 0.041 & 0.012 & 0.066 & 0.103 & 0.084 & 0.110 & 34.48 & 0.010 & 2.830 \\
\hline LB2 & 0.064 & 0.066 & 0.020 & 0.063 & 0.045 & 0.045 & 0.060 & 0.03 & 0.018 & 0.476 \\
\hline LB2 & 0.064 & 0.032 & 0.017 & 0.060 & 0.019 & 0.020 & 0.045 & 0.03 & 0.012 & 0.392 \\
\hline LB2 & 0.057 & 0.068 & 0.018 & 0.045 & 0.040 & 0.026 & 0.043 & 0.03 & 0.005 & 0.448 \\
\hline LB3 & 0.034 & 0.029 & 0.004 & 0.032 & 0.031 & 0.018 & 0.022 & 0.11 & 0.006 & 3.980 \\
\hline LB3 & 0.241 & 0.044 & 0.016 & 0.054 & 0.080 & 0.040 & 0.058 & 0.38 & 0.008 & 8.100 \\
\hline LB3 & 0.169 & 0.058 & 0.016 & 0.035 & 0.055 & 0.026 & 0.023 & 0.30 & 0.004 & 6.530 \\
\hline LB4 & 0.054 & 0.025 & 0.015 & 0.047 & 0.033 & 0.029 & 0.036 & 0.25 & 0.010 & 13.110 \\
\hline LB4 & 0.035 & 0.037 & 0.009 & 0.039 & 0.029 & 0.019 & 0.032 & 0.41 & 0.008 & 8.310 \\
\hline LB4 & 0.037 & 0.033 & 0.009 & 0.049 & 0.027 & 0.029 & 0.033 & 0.16 & 0.006 & 10.310 \\
\hline LB5 & 0.067 & 0.028 & 0.008 & 0.047 & 0.017 & 0.025 & 0.031 & 0.49 & 0.008 & 11.550 \\
\hline LB5 & 0.054 & 0.051 & 0.014 & 0.069 & 0.023 & 0.020 & 0.035 & 0.33 & 0.008 & 10.550 \\
\hline LB5 & 0.222 & 0.066 & 0.012 & 0.041 & 0.061 & 0.033 & 0.034 & 1.35 & 0.047 & 13.900 \\
\hline LB6 & 0.050 & 0.042 & 0.017 & 0.040 & 0.050 & 0.029 & 0.046 & 0.02 & 0.015 & 0.565 \\
\hline LB6 & 0.093 & 0.053 & 0.021 & 0.062 & 0.041 & 0.041 & 0.034 & 0.31 & 0.007 & 0.905 \\
\hline LB6 & 0.104 & 0.048 & 0.012 & 0.045 & 0.052 & 0.021 & 0.053 & 0.54 & 0.011 & 1.174 \\
\hline LB7 & 0.603 & 0.103 & 0.024 & 0.080 & 0.045 & 0.046 & 0.049 & 10.66 & 0.362 & 0.693 \\
\hline LB7 & 0.704 & 0.132 & 0.030 & 0.064 & 0.042 & 0.023 & 0.033 & 8.30 & 0.242 & 3.680 \\
\hline LB7 & 0.124 & 0.063 & 0.010 & 0.064 & 0.036 & 0.024 & 0.035 & 2.84 & 0.041 & 3.810 \\
\hline
\end{tabular}

